\title{
Study on the chaotic dynamics in yaw-pitch-roll coupling of asymmetric rolling projectiles with nonlinear aerodynamics
}

\author{
Yanli Xu • Baozeng Yue $\mathbb{D} \cdot$ Zhengmao Yang • \\ Liangyu Zhao · Shuxing Yang
}

Received: 18 December 2018 / Accepted: 20 July 2019 / Published online: 5 August 2019

(C) Springer Nature B.V. 2019

\begin{abstract}
To predict the coning motion forms of a rolling projectile with configurational asymmetries, the nonlinear characteristics for the system are investigated in this paper. The nonlinear dynamic model of rolling projectiles in coning motion is built by considering the nonlinear aerodynamics and roll orientation-dependent aerodynamics. The configurational asymmetry is modeled as a periodically parametric excitation in order to study its effect on the periodic response stability of the rolling projectile. Numerical continuation method is resorted to determine the parametric zone for the steady motions, and the possible stable rotational speeds are discussed. The numerical simulations, Lyapunov exponent spectrum analysis and Poincaré sections are performed to confirm the existence of chaotic coning motion. The results shown in this study not only contribute to an in-depth understanding for the nonlinear dynamics of rolling projectiles but also provide an important reference for the further study of the control
\end{abstract}

Y. Xu $\cdot$ B. Yue $(\bowtie) \cdot$ L. Zhao

School of Aerospace Engineering, Beijing Institute of Technology, Haidian District,

Beijing 100081, People's Republic of China

e-mail: bzyue@bit.edu.cn

Z. Yang

Institute of Mechanics, Chinese Academy of Sciences,

Beijing 100190, People's Republic of China

\section{S. Yang}

Institute of Xian Modern Control \& Technology, Xian 710065, Shanxi, People's Republic of China design for the yaw-pitch-roll coupling of asymmetric rolling projectiles with nonlinear aerodynamics.

Keywords Nonlinear rolling projectile - Chaos · Equilibrium · Bifurcation - Lyapunov exponent analysis $\cdot$ Routes to chaotic motion

\section{Introduction}

In engineering application, effect of manufacturing tolerances, mass distribution, external geometry and accidental damage at launch, the projectile will have different degrees of asymmetry, which may result in a coning motion caused by roll-yaw resonance phenomenon, where the nose of the body describes circles around the velocity vector, as shown in Fig. 1. The roll-yaw resonance phenomenon caused by the slight configurational asymmetries was first found by Nicolaides [1]. The resonance occurs when the roll rate is near the pitch angular frequency of the missile, which displays an amplification of the trim angle. The enlarged angle will cause a nonlinearly induced roll moment in both angle of attack and roll orientation that in some cases may lock the roll rate to the pitch frequency; this kind of motion is called lunar motion because it is similar to the motion of the moon [2]. Research by Price [3] suggested that the lateral displacement of the center of mass is the main cause of these induced roll moments. Subsequently, the occasional anomalous flight behaviors of a missile near resonance having slight configuration asymmetry have 
been widely studied by researchers. To prove the existence of steady-state resonance in free flight, a large number of authors have developed increasingly complex models. A significant research about roll lock-in phenomenon was contributed by Murphy [4]. He proposed a coupled analytical model for the problem of persistent resonance that combines linear lateral aerodynamics with nonlinear roll orientation-dependent induced moment due to center-of-mass offset. A simple graphical method was subsequently developed to determine the equilibrium points, and the corresponding stability was determined using Lyapunov's linearization method. Three different types of lock-in were shown in Murphy's work, including steady-state roll, normal persistent resonance roll, and reverse persistent resonance roll. A two-dimensional topological model was then presented by Ananthkrishnan and Raisinghani [5] to show a dependence of resonant lock-in solutions on the initial conditions; they also showed the possibility of the quasi-steady lock-in and investigated the problem of passage through resonance of rolling finned projectiles relating to the transient resonance with center of mass offset [6,7].

While studying the analytical algorithm of asymmetric rolling angle motion, the expressions of aerodynamic forces and aerodynamic moments were also continually improved to obtain more accurate prediction of dynamic characteristics. Pepitone and Jacobson [8] pointed out that any aerodynamic force or moment which is periodic in missile roll orientation may offer the potential of exciting missile resonant behavior. In his work, the cubic in angle of attack and harmonic in missile roll orientation were incorporated into the equations of angular motion. The results showed that the roll orientation-dependent restoring and side moments have a dramatic influence on missile stability at the fundamental resonance roll rate. Murphy and Mermagen [9] also confirmed that the occurrence of lock-in can be caused by a side moment that varies with the roll angle orientation in the absence of an asymmetry-induced trim moment. Based on the work of reference [9], Li et al. [10] analyzed the effect of nonlinear aerodynamic coefficients on the lock-in parameters. To obtain the desired lock-in motion, a model for optimizing some of the nonlinear aerodynamic coefficients was also constructed by them. Morote and Liaño et al. [11-14] presented a nonlinear model for the steady-state coupled pitch-yaw-rolling motion of a rocket in free flight, including nonlinear transverse aerodynamic properties and nonlinear induced moment. An effective method to avoid the occurrence of the roll lock-in was presented using numerical simulation and graphical methods. It is worth noting that the coupling between the angular motion and roll motion increases with the nonlinearities. Hence, the rolling projectile system is a complex high-dimensional dynamic system having strong nonlinear and coupling characteristics.

In recent years, the research on the nonlinear dynamics and chaos has been extended to aerospace engineering [15-18]. However, study on the chaotic dynamics in rolling projectile system is rarely found in the public literature. In fact, the aperiodic behavior observed as early as 1966 in Nicolaides's dynamic supersonic wind tunnel tests of the Aerobee sounding rocket. The studies suggested that the aperiodic behavior manifested itself due to poor roll dynamic stability and strong nonlinearities [19]. Tanrikulu [20] performed extensive numerical simulations of the analytical persistent resonance model of Murphy; the chaotic motions were also observed. Chaos is used to describe a type of motion or time evolution caused by dynamical systems. Although chaos theory has been widely studied, there is still no precise definition of chaos. However chaotic motion can be characterized by some special identifiable properties [21-25]: chaos can be defined as a bounded aperiodic behavior of deterministic nonlinear systems, which is not an equilibrium solution, a periodic solution or a quasiperiodic solution. In addition, chaotic motion is also characterized by sensitivity to initial states, that is, tiny differences in the initial conditions can be rapidly amplified to result in huge differences in the response. The numerical identification of chaos plays an important role in nonlinear dynamics to determine whether chaotic behavior occurs in a specific system. Lyapunov exponents and Lyapunov dimensions are introduced to specify the sensitivity of chaos to initial states and to emphasize the recurrent aperiodicity of chaos, respectively. Power spectra may be used to detect the stochasticity of chaos and Poincaré maps are employed to determine the geometrical structures of chaos [21,22]. From the emerging processes of chaotic motion changing with system parameters, routes to chaotic motions can also serve as an effective approach to investigate chaotic motion. In most systems, chaos occurs only within some range of parameter values. How to turn a regularly behaving system into chaos is a fundamental and significant problem. There are several fairly well-understood and relatively easily 
recognizable routes to chaos in a specific transition process of a prescribed system, including period-doubling sequence, intermittency, quasiperiodic torus breakdown, quasiperiodic torus doubling, crises, period-3 motions and so on [21-32].

This paper considers both nonlinear aerodynamics and roll orientation-dependent aerodynamics to establish a nonlinear dynamical model that can accurately describe the coning motion of rigid-body rolling projectile. Numerical continuation method and modern nonlinear dynamic theories are used to reveal the various possibilities of coning motion forms for asymmetric rolling projectile. Numerical continuation method is used to establish the critical values of the bifurcation parameters and to predict the limit cycles in the asymmetric rolling projectiles to locate the stable and unstable regions that give sufficient information for further exploring more complex nonlinear behaviors [33-36]. Also, Lyapunov exponent analysis and bifurcation diagrams are presented to reveal the dependence of coning motion forms (including chaotic motion) on critical parameters. In the literature available on the topic, three are few reports of exploring routes to chaos for this newly proposed nonlinear asymmetric rolling projectile chaotic system. The results obtained herein not only serve as new examples for the routes to chaos, geometrical structure of chaos and numerical identification of chaos but also are helpful in designing control strategy for guaranteeing stability and suppressing chaotic motion of coupled rolling projectile system in coning motion.

The rest of the paper is shown as follows. In Sect. 2, coupling pitch-yaw-roll equations of motion are presented for the rolling projectile system and the analysis of the influence of nonlinear aerodynamics on the coupled system is also included in this section. Section 3 explores the conditions for the steady motions followed by extracting critical parameter values corresponding to the different types of bifurcations. In Sect. 4, the bifurcation diagrams and Lyapunov exponent analysis are carried out to investigate the nonlinear dynamic characteristics of the rolling projectile system with variation of asymmetry effect and design roll angle rate. The routes to chaos are numerically examined via the orbit diagrams, phase trajectories, Poincaré maps and power spectra. Numerical results demonstrate that the onset of chaos is characterized by the period-doubling bifurcation as the design roll angle rate is increased. Finally, some conclusions are drawn in Sect. 5.

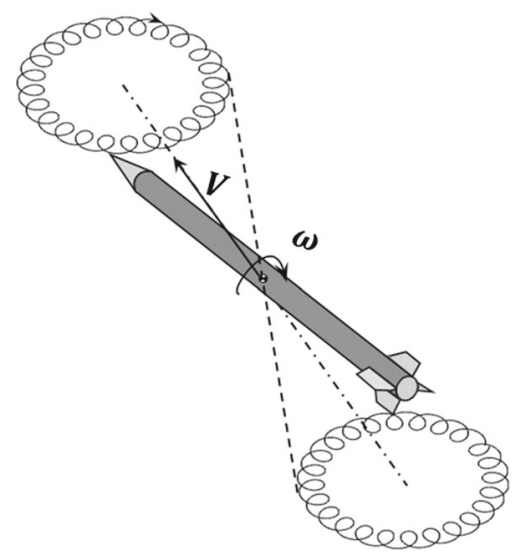

Fig. 1 Coning motion of rolling projectile

\section{Mathematical model}

\subsection{Construction of lateral dynamical model}

A spinning missile is a kind of air vehicle that rolls around its longitudinal axis continuously during flight. It can be observed from the dynamic process of the angular motion that, besides the rotation about its longitudinal axis, there exists another form of circular motion, known as coning motion of longitudinal axis around the velocity vector. The rotation of a missile induces a serious coupling between the pitch and the yaw channels, and non-convergence motion may occur if the deviation between the actual nutation angle and the design nutation angle increases continuously or maintains comparatively large amplitude.

A body-fixed coordinate system $(O-x y z)$ is introduced with $x$ axis which is the longitudinal axis of missile, $y$ axis is in the plane of a fin, and $z$ axis determined by the right-hand rule is orthogonal to the $x$ axis and $y$ axis. Let $\boldsymbol{i}, \boldsymbol{j}, \boldsymbol{k}$ be the basis vectors of $(O-x y z)$ and $u, v, w$ be the components of velocity vector for the missile's center of mass and $p, q, r$ be the components of angular velocity vector. Then the vectors $\boldsymbol{V}$ and $\boldsymbol{\omega}$ can be written as :

$\boldsymbol{V}=u \boldsymbol{i}+v \boldsymbol{j}+w \boldsymbol{k}, \quad \boldsymbol{\omega}=p \boldsymbol{i}+q \boldsymbol{j}+r \boldsymbol{k}$

The dynamical equations can be derived from Newton's second law and theorem of moment of momentum, when the dynamical equations are expressed in $(O-x y z)$, it yields [37] 


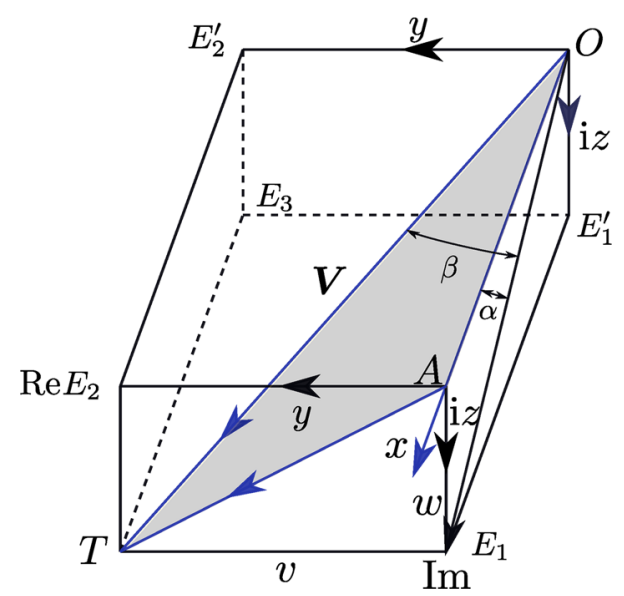

Fig. 2 Definition of complex variables

$$
\left\{\begin{array}{l}
\frac{\mathrm{d} v}{\mathrm{~d} t}+u r-p w=\frac{F_{y}}{m} \\
\frac{\mathrm{d} w}{\mathrm{~d} t}-u q+p v=\frac{F_{z}}{m} \\
\frac{\mathrm{d} q}{\mathrm{~d} t}-p r(1-\sigma)=\frac{M_{y}}{I_{t}} \\
\frac{\mathrm{d} r}{\mathrm{~d} t}+p q(1-\sigma)=\frac{M_{z}}{I_{t}}
\end{array}\right.
$$

where $\sigma=I_{x} / I_{t}$, and $I_{x}, I_{t}$ represent axial moment of inertia and transverse moment of inertia; $m$ is the mass of the projectile; $F_{y}, F_{z}, M_{y}, M_{z}$, respectively, denote the projections of aerodynamic force and aerodynamic moment on $y$ axis and $z$ axis.

To avoid the small angle hypothesis and the inconvenience caused by the introduction of a large number of trigonometric and inverse trigonometric functions in the process of modeling, the variation of body axis relative to the velocity vector is directly reflected by the components of $v / V, w / V$. As shown in Fig. 2, the $(A-y z)$ plane is defined as a complex plane, the complex angle of attack and the complex transverse angular velocity can be defined as

$\xi=(v+\mathrm{i} w) / V=|\xi| \mathrm{e}^{\mathrm{i} \theta}, \quad \mu=(q+\mathrm{i} r) d / V$

where $\theta$ specifies the orientation of the plane of complex angle of attack with respect to the $(A-x y)$ plane, $d$ is diameter of projectile, $V$ is magnitude of the missile velocity, $\mathrm{i}$ is imaginary unit, and $\mathrm{e}$ is natural constant.

The aerodynamic force and aerodynamic moment in $(O-x y z)$ can be written as complex function of angle of attack, that is $[9,38]$

$$
\begin{aligned}
F_{y}+\mathrm{i} F_{z}= & -\frac{\rho V^{2} S}{2} C_{N \alpha} \xi-\frac{\rho V^{2} S}{2} C_{N 0} \mathrm{e}^{\mathrm{i} \phi_{N}} \\
M_{y}+\mathrm{i} M_{z}= & \frac{\rho V^{2} S d}{2}\left[\frac{p d}{V}\left(C_{M p \alpha 0}+C_{M p \alpha 2} \xi \bar{\xi}\right) \xi\right. \\
& -\mathrm{i}\left(C_{M \alpha 0}+C_{M \alpha 2} \xi \bar{\xi}+C_{M R \theta} \xi \bar{\xi} \mathrm{e}^{-\mathrm{i} 4 \theta}\right) \xi \\
& -\mathrm{i}\left(C_{M \dot{\alpha} 0}+C_{M \dot{\alpha} 2} \xi \bar{\xi}\right)(\dot{\xi}+\mathrm{i} p \xi)(d / V) \\
& \left.+\left(C_{M q 0}+C_{M q 2} \xi \bar{\xi}\right) \mu-\mathrm{i} C_{M 0} \mathrm{e}^{\mathrm{i}\left(\phi_{M}\right)}\right]
\end{aligned}
$$

where $\rho$ is air density, $S=\pi d^{2} / 4$ is reference area of projectile, $C_{N \alpha}$ is angle of attack coefficient of the normal force, $C_{N 0}$ and $\phi_{N}$, respectively, represent asymmetry force coefficient and asymmetry force orientation angle, $C_{M p \alpha 0}$ and $C_{M p \alpha 2}$, respectively, represent linear and nonlinear angle of attack coefficients of Magnus moment, $C_{M \alpha 0}$ and $C_{M \alpha 2}$, respectively, represent linear and nonlinear angle of attack coefficients of static moment, $C_{M q 0}, C_{M \dot{\alpha} 0}, C_{M q 2}, C_{M \dot{\alpha} 2}$ are linear and nonlinear damping and lag coefficients of the pitch moment, $\phi_{M}$ and $C_{M 0}$ are asymmetry moment orientation angle and coefficient of asymmetry moment, $C_{M R \theta}$ is roll orientation-dependent static moment coefficient, and $\bar{\xi}$ represents the complex conjugate of $\xi$.

Introducing dimensionless arc length $s$, namely, $s=$ $d^{-1} \int_{0}^{t} V \mathrm{~d} t$, and then Eq. (2) can be rewritten as

$$
\left\{\begin{array}{l}
\frac{v^{\prime}}{V}+\frac{u}{V}\left(\frac{r d}{V}\right)-\frac{w}{V}\left(\frac{p d}{V}\right)=\frac{F_{y}}{m}\left(\frac{d}{V^{2}}\right) \\
\frac{w^{\prime}}{V}-\frac{u}{V}\left(\frac{q d}{V}\right)+\frac{v}{V}\left(\frac{p d}{V}\right)=\frac{F_{z}}{m}\left(\frac{d}{V^{2}}\right) \\
\frac{q^{\prime} d}{V}-\left(\frac{p d}{V}\right) \cdot\left(\frac{r d}{V}\right)(1-\sigma)=\frac{M_{y}}{I_{t}}\left(\frac{d}{V}\right)^{2} \\
r^{\prime} \frac{d}{V}+\left(\frac{p d}{V}\right) \cdot\left(\frac{q d}{V}\right)(1-\sigma)=\frac{M_{z}}{I_{t}}\left(\frac{d}{V}\right)^{2}
\end{array}\right.
$$

where the tilde superscript represents differentiation with respect to the dimensionless arc length $s$. Also

$\frac{\mathrm{d} V}{\mathrm{~d} t}=V^{\prime} \frac{V}{d}=-\frac{\rho V^{2} S C_{D}}{2 m}$

where $C_{D}=C_{D 0}+C_{D 2} \xi \bar{\xi}$ is the drag force coefficient.

Multiplying the second and the fourth equations of Eq. (6) by imaginary number $i$ and adding to the first and the third equations of Eq. (6), respectively, and using Eqs. (3)-(5) and (7), the nonlinear second-order differential equation in the complex angle of attack can be expressed as in the following: 


$$
\begin{aligned}
& \xi^{\prime \prime}+\left[H-\mathrm{i}(\sigma-2) \phi^{\prime}\right] \xi^{\prime} \\
& +\left[(1-\sigma)\left(-\phi^{\prime 2}\right)-M-M_{R \theta}\right. \\
& \left.\quad+\mathrm{i}\left(\phi^{\prime \prime}+\phi^{\prime} h\right)\right] \xi=A k_{t}^{-2} C_{M 0} \mathrm{e}^{\mathrm{i} \phi_{M}}
\end{aligned}
$$

where $\phi$ is roll angle, and $\phi^{\prime}=p d / V$. Also

$$
\begin{aligned}
H= & H_{0}+H_{2}, \quad T=T_{0}+T_{2}, \quad M=M_{0}+M_{2}, \\
h= & H-\sigma T, M_{R \theta}=C_{M R \theta} \xi \bar{\xi} \mathrm{e}^{-\mathrm{i} 4 \theta} \\
H_{0}= & A\left[C_{N \alpha 0}-2 C_{D 0}-k_{t}^{-2}\left(C_{M q 0}\right.\right. \\
+ & \left.\left.C_{M \dot{\alpha} 0}\right)\right], \quad M_{0}=A k_{t}^{-2} C_{M \alpha 0}, \\
T_{0}= & A\left(C_{N \alpha 0}-C_{D 0}+k_{t}^{-2} C_{M p \alpha 0}\right) \\
H_{2}= & A\left[C_{N \alpha 2}-2 C_{D 2}-k_{t}^{-2}\left(C_{M q 2}\right.\right. \\
& \left.\left.+C_{M \dot{\alpha} 2}\right)\right] \xi \bar{\xi}, \quad M_{2}=A k_{t}^{-2} C_{M \alpha 2} \xi \bar{\xi}, \\
T_{2}= & A\left(C_{N \alpha 2}-C_{D 2}+k_{t}^{-2} C_{M p \alpha 2}\right) \xi \bar{\xi}, \\
A= & \rho S d / 2 m, k_{t}^{2}=I_{t} / m d^{2}
\end{aligned}
$$

Linear flight dynamics show that the frequency of the motion is decided by $M_{0}$. Introduction of the second dimensionless time $\tau=\left[\left|M_{0}\right| /(1-\sigma)\right]^{1 / 2} s$ in Eq. (8) leads to

$$
\begin{aligned}
\ddot{\xi}+ & {[\hat{H}-\mathrm{i}(\sigma-2) \dot{\phi}] \dot{\xi}+\left[(1-\sigma)\left(1-\dot{\phi}^{2}\right)-\hat{M}_{2}\right.} \\
& \left.-\hat{M}_{R \theta}+\mathrm{i}(\ddot{\phi}+\dot{\phi} \hat{h})\right] \xi=\frac{A k_{t}^{-2}(1-\sigma)}{M_{0}} C_{M 0} \mathrm{e}^{\mathrm{i} \phi_{M}}
\end{aligned}
$$

where the dot represents differentiation with respect to $\tau$, and

$$
\begin{gathered}
\hat{h}=h\left[(1-\sigma) /\left|M_{0}\right|\right]^{1 / 2}, \\
\hat{M}_{2}=M_{2}\left[(1-\sigma) /\left|M_{0}\right|\right], \\
\hat{M}_{R \theta}=M_{R \theta}\left[(1-\sigma) /\left|M_{0}\right|\right]
\end{gathered}
$$

According to Eq. (9), resonance occurs at $|\dot{\phi}|=1$.

The equilibrium points are found by setting $\ddot{\xi}=\dot{\xi}=$ $\ddot{\phi}=0$. Meanwhile writing the complex angle of attack in polar coordinates, Eq. (9) can be rewritten as:

$$
\begin{aligned}
& {\left[(\sigma-1) \dot{\phi}_{e}^{2}-\hat{M}_{0}-\hat{M}_{2} \delta_{e}^{2}-\hat{C}_{M R \theta} \delta_{e}^{2} \mathrm{e}^{-\mathrm{i} 4\left(\phi_{M}-\varphi_{e}\right)}\right.} \\
& \left.\quad+\mathrm{i}\left(\dot{\phi}_{e}\left(\hat{h}_{0}+\hat{h}_{2} \delta_{e}^{2}\right)\right)\right] \delta_{e} \mathrm{e}^{-\mathrm{i} \varphi_{e}} \\
& \quad=\frac{A k_{t}^{-2}(1-\sigma)}{\hat{M}_{0}} C_{M 0}
\end{aligned}
$$

where ()$_{e}$ represents equilibrium value, $\delta$ is the absolute value of $\xi$ and $\bar{\xi}, \varphi$ is phase lag.

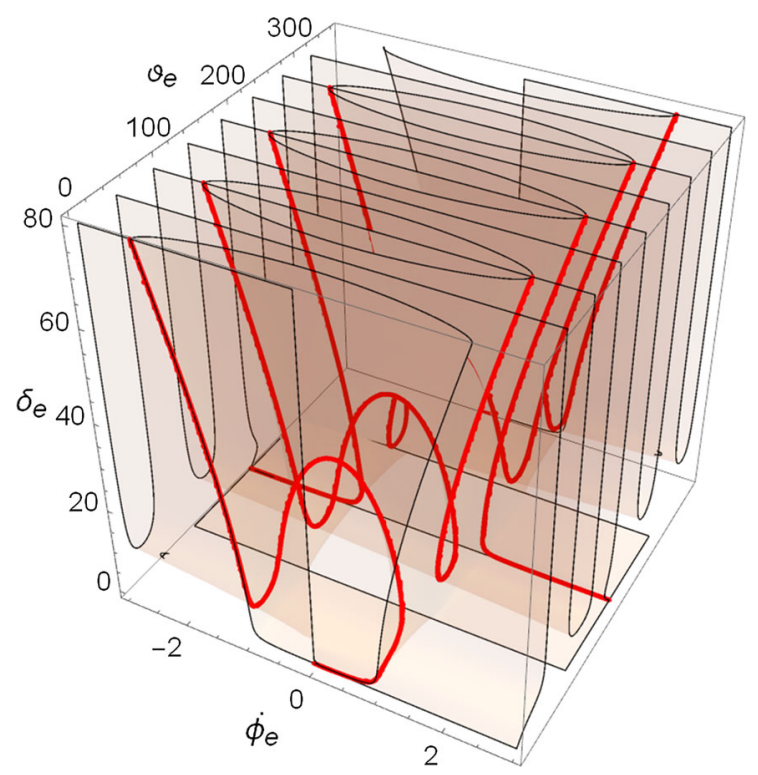

Fig. 3 Steady-state lateral equilibrium points

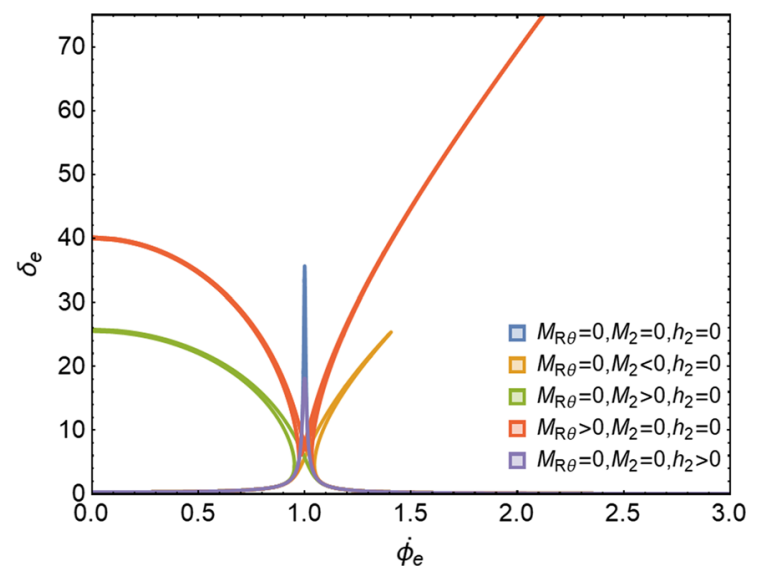

Fig. 4 Comparison of frequency responses

Figure 3 gives a discontinuous curve intersection of two surfaces composed of the real and imaginary parts of Eq. (10). For comparison analysis, Fig. 4 gives an amplitude-frequency response which is a projection of the curve intersection of Fig. 3 onto the $\delta_{e}-\dot{\phi}_{e}$. The relative parameters are shown in Table 1.

In Fig. 4, it can be observed that the resonant frequency of the system with $h_{2}$ is similar as that of the system without $h_{2}$, only showing a difference at amplitude. The existence of $M_{2}$ bends the frequency response curve to one side; if $M_{2}>0$, it bends the whole curve to the left, acting as a "soft-spring"; if $M_{2}<0$, it bends 
Table 1 Physical and aerodynamic parameters [39]

\begin{tabular}{llllll}
\hline Parameters & Values & Unit & Parameters & Values & Unit \\
\hline$\sigma$ & 0.05 & - & $M_{R \theta}$ & $-2 \times 10^{-5}$ & - \\
$\rho$ & 1.03 & $\mathrm{~kg} / \mathrm{m}^{3}$ & $C_{M 0}$ & 0.03 & - \\
$S$ & $1.27 \times 10^{-2}$ & $\mathrm{~m}^{2}$ & $\phi_{M}$ & 90 & $\circ$ \\
$d$ & 0.127 & $\mathrm{~m}$ & $M_{0}$ & $-9.78 \times 10^{-6}$ & - \\
$m$ & 30 & $\mathrm{~kg}$ & $M_{2}$ & $\pm 4.88 \times 10^{-5}$ & - \\
$V$ & 280 & $\mathrm{~m} / \mathrm{s}$ & $h_{0}$ & $1.0 \times 10^{-2}$ & - \\
$k_{t}^{-2}$ & 0.059 & - & $h_{2}$ & $2.60 \times 10^{-4}$ & - \\
\hline
\end{tabular}

the whole curve to the right, acting as a "hard-spring". Figure 4 also shows that the presence of $M_{R \theta}$ causes the single amplitude lobe to be divided into two tongues for the roll orientation-dependent curves. The left tongue acts as "soft-spring" and the right tongue acts as "hardspring". Meanwhile, $M_{R \theta} \neq 0$ also makes possible the existence of very large amplitudes as shown in this figure. Results mentioned above show that the nonlinear aerodynamic moments $M_{2}$ and $M_{R \theta}$ have significant influence on the periodic response of the rolling projectile.

\subsection{Roll equation of motion}

The roll moment considered here has two components: a constant driving moment created by differentially canting the fins to control the missile, and a spindamping moment proportional to the roll rate:

$M_{x}=\frac{\rho V^{2} S d}{2}\left[C_{l p}(p d / V)+C_{l 0}\right]$

Here $C_{l p}$ is spin-damping moment coefficient and $C_{l 0}$ is spin-producing moment coefficient created by canting the fins.

Zero roll moment causes the missile to fly at a constant-steady-state spin, i.e.,

$C_{l 0}=-p_{\mathrm{ss}} d C_{l p} / V$

where $p_{\mathrm{ss}}$ is constant-steady-state spin.

The complete roll moment including a nonlinear induced roll moment term can be rewritten as:

$M_{x}=\frac{\rho V^{2} S d}{2}\left[C_{l p}\left(p-p_{\mathrm{ss}}\right)(d / V)+C_{l \theta}(\delta, \theta)\right]$

Here, $C_{l \theta}$ is the induced rolling moment coefficient and it is an odd function of $\theta$ with a period of $2 \pi / n$. $C_{l \theta}(\theta, \delta)$ can be expanded as the following Fourier series only considering fundamental wavelength:

$$
\begin{aligned}
C_{l \theta} & =\sum_{k=1} \sum_{h=0} a_{k h} \delta^{k n} \delta^{h} \sin n \theta \\
& =\sum_{k=1} \sum_{h=0} a_{k h} \delta^{k n} \delta^{h} \frac{\xi^{k n}-\bar{\xi}^{k n}}{2 \mathrm{i} \delta^{k n}} \\
& =\sum_{k=1} \sum_{h=0} a_{k h} \delta^{h} \frac{\xi^{k n}-\bar{\xi}^{k n}}{2 \mathrm{i}}
\end{aligned}
$$

The roll equation of motion can be obtained by differentiating the axial angular momentum and equating them to roll moment $M_{x}$. The roll equation can be obtained as follows when only considering the first term for the induced roll moment.

$\phi^{\prime \prime}+K_{p}\left[\phi^{\prime}-\phi_{\mathrm{ss}}^{\prime}-\mathrm{i} K_{\theta}\left(\xi^{n}-\bar{\xi}^{n}\right)\right]=0$

where $\phi_{\mathrm{ss}}^{\prime}=p_{\mathrm{ss}} d / V, K_{p}=-A\left(k_{x}^{-2} C_{l p}-C_{D}\right)$ and $k_{x}^{-2}=m d^{2} / I_{x}$. The independent variable in Eq. (15) is dimensionless arc length $s$. Introduction of $\tau=$ $\left[\left|M_{0}\right| /(1-\sigma)\right]^{1 / 2} s$ into Eq. (15) yields

$\ddot{\phi}+\hat{K}_{p}\left[\dot{\phi}-\dot{\phi}_{\mathrm{ss}}-\mathrm{i} \hat{K}_{\theta}\left(\xi^{n}-\bar{\xi}^{n}\right)\right]=0$

where $\hat{K}_{p}=K_{p}\left[-(1-\sigma) / M_{0}\right], \hat{K}_{\theta}=K_{\theta}$ $\left[-(1-\sigma) / M_{0}\right]$.

\section{Equilibrium and bifurcation analysis for spin-yaw lock-in}

In order to analyze the equilibrium behavior of projectiles, Murphy [4] firstly proposed a graphical method to locate equilibrium points and then this method was used in the subsequent studies $[5,20]$. However, for the projectile dynamics with high dimensions and strong nonlinearities, this method is no longer applicable. To analyze the local stability for different asymmetry moment orientation angles, a numerical continuation method [33-36] is applied to determine the equilibrium behavior of system with varying parameters. The numerical 
Table 2 Computed bifurcation points

\begin{tabular}{lllll}
\hline No\# & $\phi_{M}\left(^{\circ}\right)$ & TY & $a$ & $(\beta+\mathrm{i} \alpha, \dot{\phi})$ \\
\hline 1 & 3.22 & $\mathrm{LP}$ & 0.045 & $(-5.50-\mathrm{i} 0.50,1.06)$ \\
2 & 28.18 & $\mathrm{NE}$ & - & $(-0.83-\mathrm{i} 0.48,1.14)$ \\
3 & 28.20 & $\mathrm{LP}$ & -0.271 & $(-3.28-\mathrm{i} 1.04,1.05)$ \\
4 & 140.59 & $\mathrm{H}$ & -0.094 & $(1.16-\mathrm{i} 1.05,-1.09)$ \\
5 & $\mathrm{LP}$ & 0.153 & $(2.96-\mathrm{i} 0.50,1.06)$ \\
6 & $\mathrm{H}$ & 0.138 & $(5.63-\mathrm{i} 1.04,-1.06)$ \\
7 & 163.25 & $\mathrm{LP}$ & 0.152 & $(4.41-\mathrm{i} 1.04,-1.05)$ \\
8 & 163.65 & $\mathrm{H}$ & -0.002 & $(0.03+\mathrm{i} 0.01,3.05)$ \\
9 & 165.15 & $\mathrm{NE}$ & - & $(-1.16-\mathrm{i} 1.00,-0.90)$ \\
10 & 203.01 & $\mathrm{H}$ & -0.989 & $(1.05-\mathrm{i} 1.00,-0.90)$ \\
11 & 218.47 & $\mathrm{H}$ & -0.005 & $(1.04-\mathrm{i} 0.55,0.87)$ \\
12 & 228.22 & $\mathrm{H}$ & -0.129 & $(-0.03+\mathrm{i} 0.01,3.05)$ \\
13 & 314.46 & $\mathrm{H}$ & -0.008 & $(4.36-\mathrm{i} 1.02,-0.99)$ \\
14 & 334.20 & $\mathrm{H}$ & 0.020 & \\
\hline
\end{tabular}

$T Y$ type of point, $L P$ limit point, $H$ Hopf bifurcation point, $N E$ Neutral saddle equilibrium. $a$ is quadratic normal form coefficient for fold bifurcation or the first Lyapunov coefficient for Hopf bifurcation

Fig. 5 Location of equilibrium with nonlinear aerodynamics

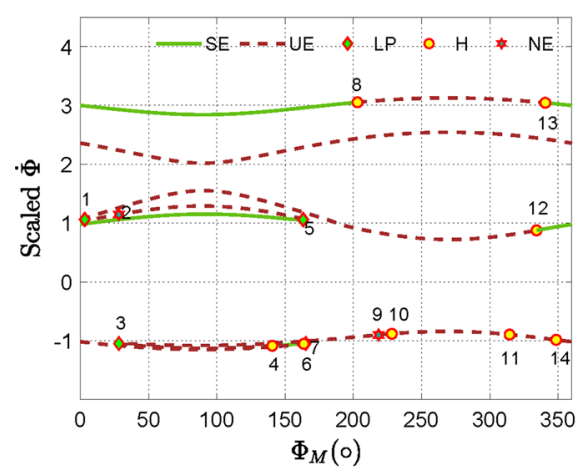

(a)

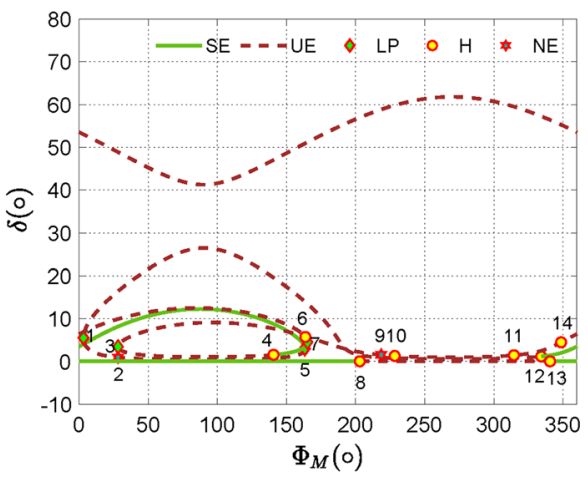

(b) results obtained by using continuation method for the bifurcation value of system parameters are shown as follows (Table 2).

One way to view the angular motion of this rolling projectile is to consider the responses of angle of attack $\alpha$ and angle of sideslip $\beta$. As shown in Fig. 2, $\alpha$ and $\beta$ can be calculated as

$$
\left\{\begin{array}{l}
\beta=\arcsin (v / V), \\
\alpha=\arctan \left((w / V) / \sqrt{1-\left((v / V)^{2}+(w / V)^{2}\right)}\right)
\end{array}\right.
$$

Figure 5 shows locations of possible equilibrium with nonlinear aerodynamics in terms of $\dot{\phi}$ and $\delta$. Sta- ble solutions in figures are indicated by full green lines and unstable solutions by dashed red lines. One or two or three stable fixed points may be obtained according to the value of the asymmetry moment orientation angle. The first one corresponds to the design state, and the other two correspond to the normal lock-in and reverse lock-in. As shown in Fig. 5, the number and value of the equilibrium points change with $\phi_{M}$. Cases for $0^{\circ}-140.59^{\circ}$ and $340.63^{\circ}-360^{\circ}$ show a stable design solution and a stable normal lock-in solution; case for $140.59^{\circ}-163.25^{\circ}$ shows three stable solutions: a stable design solution, a stable normal lock-in solution and a stable reverse lock-in solution; case for $163.25^{\circ}-165.15^{\circ}$ shows a stable design solution and a stable reverse lock-in solution; case $334.20^{\circ}-340.63^{\circ}$ 
only shows a stable normal lock-in solution; case for $165.15^{\circ}-203.01^{\circ}$ only shows a stable design solution, while case for $203.01^{\circ}-334.20^{\circ}$ shows no stable solutions.

It also can be observed from Fig. 5a that there is a critical value $\phi_{M}$ at which stable lock-in solutions do not occur, which are created at the limit points. When a stable resonance solution exists, there also will be unstable solutions that are very close to the lock-in solutions. These unstable solutions will play an important role in the process of passing resonance. Meanwhile, when stable lock-in occurs, the amplitude of the equilibrium nutation angle becomes larger due to the resonance effect, as illustrated in Fig. 5b. Note that the induced rolling moment caused by the equilibrium nutation angle also suppresses the tendency of the missile reaching the design rotation state. Under this interaction between rolling moment and nutation motion, equilibrium nutation angle becomes bigger.
For the cases with more than one equilibrium point, the steady-state solution depends on the initial conditions at launch. Figure 6a shows three stable equilibrium points: a stable design solution, a stable normal lock-in solution and a stable reverse lock-in solution when asymmetry moment orientation angle is $150^{\circ}$. It may be also of interest to know the range of occurrence of each equilibrium solutions for a set of realistic initial conditions. Therefore, numerical simulations are carried out with different initial conditions; the effect of disturbances at launch is given in terms of different complex angles, with all of rates assumed to be zero initially. Figure 6b illustrates the simulation results, where SDS, SNS, US and SRS represent the stable design solutions, stable normal lock-in, unstable solutions and stable reverse lock-in, respectively. It may be observed that the range of occurrence for stable reverse lock-in is rather small and design solutions and normal lock-in can be obtained easily.
Fig. 6 Steady-state solutions and the corresponding basins of attraction

Fig. 7 Bifurcation diagrams $\left(C_{M 0}=0.01\right): \mathbf{a}$ angle of attack $\alpha$ and $\mathbf{b}$ angle of sideslip $\beta$

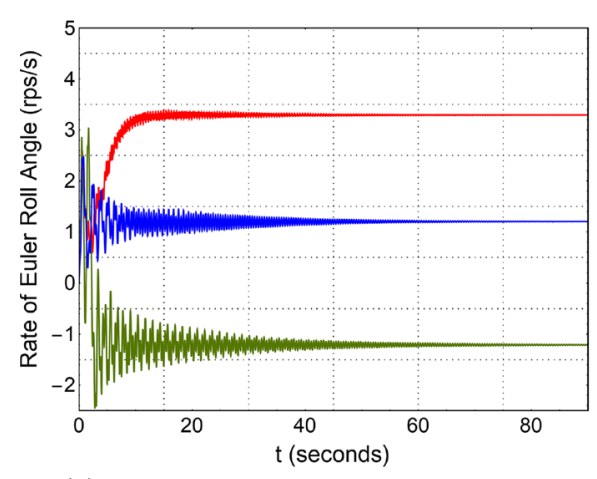

(a) Steady state solutions for intial complex angles $3^{\circ}+\mathrm{i} 1^{\circ}, 1^{\circ}+\mathrm{i} 3^{\circ}$ and $4^{\circ}+\mathrm{i} 4^{\circ}\left(\phi_{M}=150^{\circ}\right)$

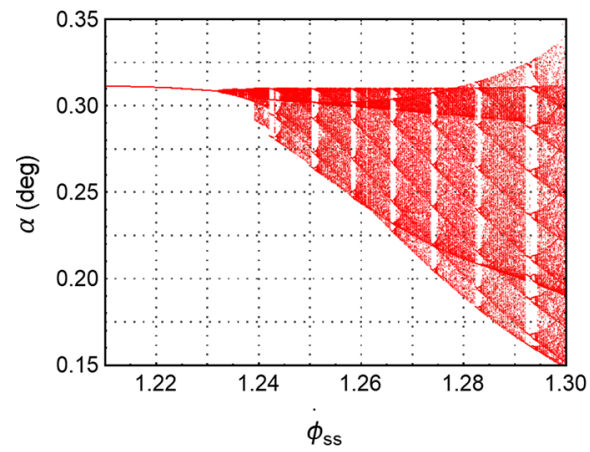

(a)

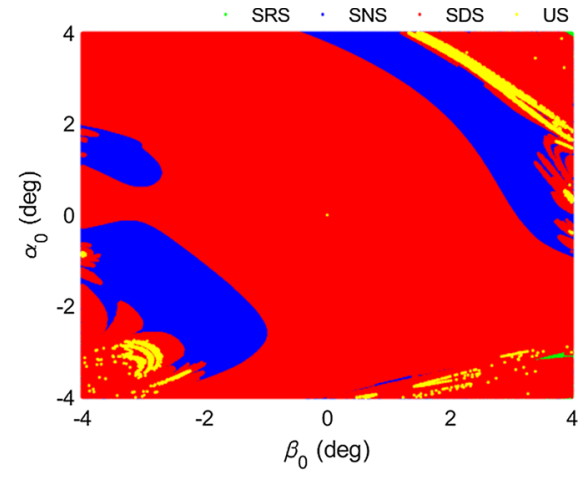

(b) The basing of attraction

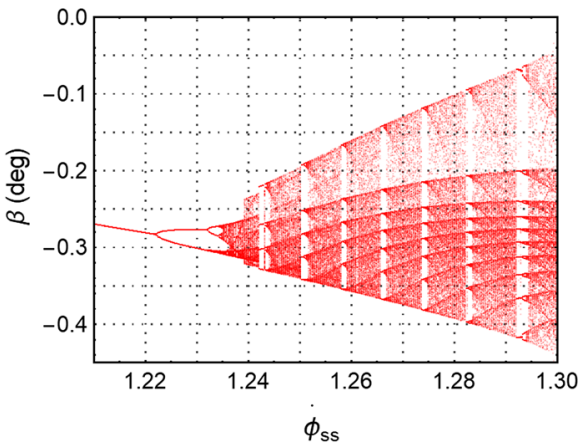

(b) 
Fig. 8 Bifurcation diagrams $\left(C_{M 0}=0.03\right)$ : $\mathbf{a}$ angle of attack $\alpha$ and $\mathbf{b}$ angle of sideslip $\beta$

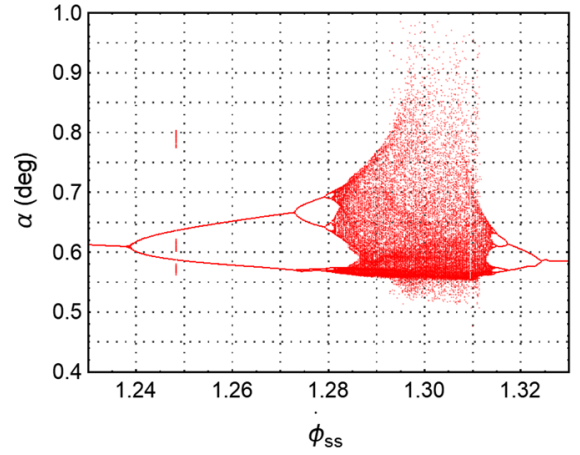

(a)

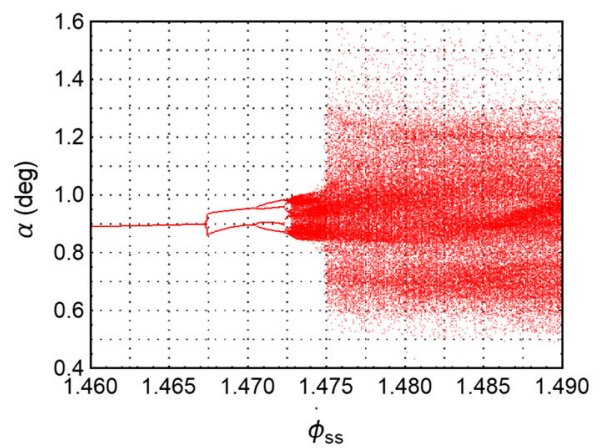

(a)

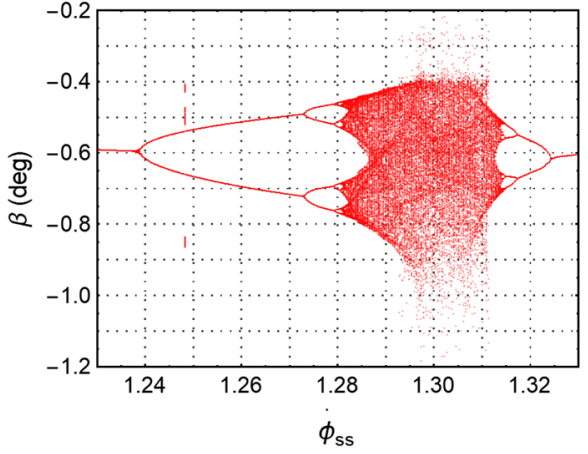

(b)

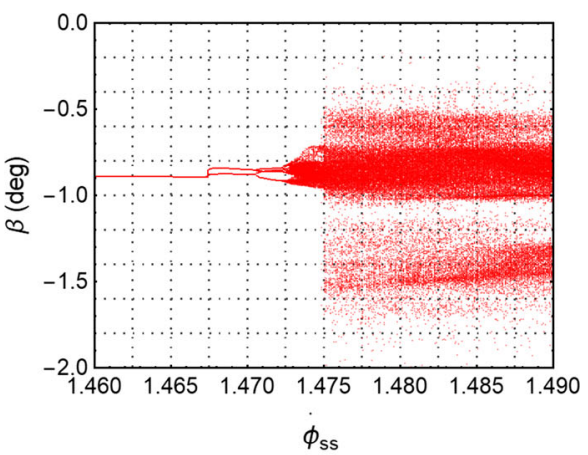

(b)

\section{Chaotic dynamics and numerical investigations}

\subsection{Effect of the asymmetry moment}

From the equilibrium analysis above, it is understood that for sufficiently large $\phi_{M}$, the equilibrium position would become unstable, and the system starts oscillating in the unstable position and eventually reaches a new stable dynamic equilibrium, i.e., limit cycle. In order to investigate the aperiodic behavior another parameter, i.e., design roll angle rate $\dot{\phi}_{\mathrm{ss}}$ has to be taken into account. For numerical example, $\phi_{M}$ is assumed to be $200^{\circ}$, and the asymmetry moment coefficient $C_{M 0}$ is chosen at different particular values. The bifurcation diagrams with the bifurcation parameter $\dot{\phi}_{\mathrm{ss}}$ are shown in Figs. 7, 8 and 9. Figure 7 shows the bifurcation diagrams for $C_{M 0}=0.01$, in which the complex nonlinear dynamic behaviors such as period-doubling bifurcation and chaotic motion of the system in the case of larger $\dot{\phi}_{\text {ss }}$ can be observed. As illustrated in Fig. 7, after $\dot{\phi}_{\mathrm{ss}}=1.235$, the final state value of the iteration has been aperiodic and the chaotic motion appeared. After entering chaos through period-doubling routes, some period windows and unstable periodic motions can still be found in different regions, indicating that there exist orders in chaos. Figure 7 also clearly shows a graph interwoven with orders and randomness, which also shows that chaos has some hierarchical structure.

Similarly, Fig. 8 for $C_{M 0}=0.03$ and Fig. 9 for $C_{M 0}=0.05$ show the bifurcation diagrams to illustrate the influence of different asymmetry moment coefficients on the dynamic characteristics of the rolling projectile system. From Figs. 7, 8 and 9, it can be seen that at different values of $\dot{\phi}_{\mathrm{ss}}$ when $C_{M 0}$ are fixed at particular constant value, the coning motion of rolling projectile may become irregular and turns into chaotic motion, and the region of period-doubling motion and chaotic motion is broadened and the amplitude is enlarged as the value of parameter $C_{M 0}$ increases.

\subsection{Chaotic dynamics diagnose using Lyapunov exponent}

The Lyapunov exponents and Lyapunov dimension play a crucial role in specifying the sensitivity of chaotic motion to initial states, which is developed as a quantitative measure to characterize the average 
exponential rate of convergence or divergence among the adjacent trajectories in state space [21-25,40-45]. By judging whether there exist largest Lyapunov exponents greater than zero, it is possible to decide whether or not there exists a dynamical chaos.

The Lyapunov exponents of this paper are calculated by the definition and the orthogonal method is resorted in the numerical procedure. Consider system (9) and (16) governed by

$\dot{\boldsymbol{x}}=\boldsymbol{f}(\boldsymbol{x} ; \mu), \boldsymbol{x} \in \mathrm{R}^{5}$

the averaged exponential divergence or convergence of unperturbed trajectory $L_{0}$ and perturbed trajectory $L_{1}$, starting in arbitrary close initial conditions $\boldsymbol{x}_{0}$ and $\boldsymbol{x}_{0}+\Delta \boldsymbol{x}_{0}$, respectively, is given by $[21,22]$

$\lambda\left(\boldsymbol{x}_{0}, \boldsymbol{e}_{0}\right)=\lim _{t \rightarrow \infty} \frac{1}{t} \ln \frac{\left\|\boldsymbol{e}\left(\boldsymbol{x}_{0}, t\right)\right\|}{\left\|\boldsymbol{e}^{0}\right\|}$

where $\boldsymbol{x}$ is the vector of state variables, $\mu$ is design roll angle rate; \|\| denotes a vector norm, $\boldsymbol{e}\left(\boldsymbol{x}_{0}, t\right)=$ $\boldsymbol{x}\left(\boldsymbol{x}_{0}+\Delta \boldsymbol{x}_{0}, t\right)-\boldsymbol{x}\left(\boldsymbol{x}_{0}, t\right)$ and $\boldsymbol{e}^{0}=\boldsymbol{e}\left(\boldsymbol{x}_{0}, 0\right)$; In the five-dimensional state space, take a set of base vectors $\left(\boldsymbol{\eta}_{i}, i=1,2, \ldots, 5\right)$, each base vector $\boldsymbol{\eta}_{i}$ corresponds to $\lambda_{i}$. These Lyapunov exponents are sorted in decreasing order such that $\lambda_{1}>\lambda_{2}>\cdots>\lambda_{5}$. The asymptotic quantity $\lambda_{i}$ given by Eq. (19) is called one-dimensional exponent, in order to calculate all of Lyapunov exponents, it has been generalized to higherdimensional Lyapunov exponents $[22,43,44]$, and the orthogonal method is applied.

For a given parallelepiped $\boldsymbol{E}^{0}=\left[\boldsymbol{e}_{1}^{0}, \ldots, \boldsymbol{e}_{p}^{0}\right]$ in $\mathrm{R}^{5}$, the Gram-Schmidt orthogonal procedure in $\boldsymbol{E}^{0}$ produces a orthogonal parallelepiped $\boldsymbol{V}^{0}$. After time $T, \boldsymbol{V}^{0}$ evolves to parallelepiped $\boldsymbol{E}^{1}=\left[\boldsymbol{e}_{1}^{1}, \ldots, \boldsymbol{e}_{p}^{1}\right]$ centered in $\boldsymbol{x}_{1}\left(\boldsymbol{x}_{0}, T\right)$. Gram-Schmidt procedure of $\boldsymbol{E}^{1}$ results in the matrix of orthonormal vectors $V^{1}=\left[v_{1}^{1}, \ldots, v_{p}^{1}\right]$, and then the parallelepiped $\boldsymbol{V}^{1}$ evolves to parallelepiped $\boldsymbol{E}^{2}=\left[\boldsymbol{e}_{1}^{2}, \ldots, \boldsymbol{e}_{p}^{2}\right]$ centered in $\boldsymbol{x}_{2}\left(\boldsymbol{x}_{1}, T\right)$ after another time $T$. Repetition of the orthogonalization and evolution $k$ times yields $[43,44]$

$$
\begin{gathered}
\lambda_{p}\left(x_{0}, \boldsymbol{E}^{0}\right)=\lim _{t \rightarrow \infty} \frac{1}{t} \ln \frac{\left\|\operatorname{Vol}^{p}\left(\boldsymbol{E}^{t}\right)\right\|}{\left\|\operatorname{Vol}^{p}\left(\boldsymbol{E}^{0}\right)\right\|} \\
=\sum_{i=1}^{p} \lambda_{i}=\lim _{k \rightarrow \infty} \frac{1}{k T} \sum_{i=1}^{k} \ln \left\|\prod_{j=1}^{p} w_{j}^{i}\right\|
\end{gathered}
$$

where $\mathrm{Vol}^{p}$ is the p-dimensional volume, $\boldsymbol{w}_{j}^{i}$ is the $j$ th orthogonal vector computed from $\boldsymbol{E}^{i}$. The definition of Eq. (20) means the mean rate of the expansion or contraction for a p-dimensional parallelepiped along the trajectory $\boldsymbol{x}\left(\boldsymbol{x}_{0}, t\right)$. The Lyapunov spectrum can be calculated as

$\lambda_{1} \approx \frac{1}{k T} \sum_{i=1}^{k} \ln \left\|\boldsymbol{w}_{1}^{i}\right\|, \ldots, \lambda_{5} \approx \frac{1}{k T} \sum_{i=1}^{k} \ln \left\|\boldsymbol{w}_{5}^{i}\right\|$

For a chaos, a positive Lyapunov exponent means that no matter how small the distance between the initial two trajectories is in phase space, the difference will increase exponentially over time and become unpredictable. In rolling projectile system, there are five Lyapunov exponents $\lambda_{1}, \lambda_{2}, \lambda_{3}, \lambda_{4}, \lambda_{5}$. The first Lyapunov exponent $\lambda_{1}$, corresponding to the most unstable direction of the flow, is measured by the largest Lyapunov exponent. The fifth Lyapunov exponent $\lambda_{5}$ describes the contracting nature of phase portraits, which must be negative. Therefore, for five-dimensional dynamical system in this paper, if all Lyapunov exponents are negative, the coning motion of rolling projectile is in a stable equilibrium; if there is a zero Lyapunov exponent and other Lyapunov exponents are negative, the coning motion of system is a stable limit cycle; if there are two or three Lyapunov exponents that are very close to zero, the coning motion is a 2-torus or 3-torus quasiperiodic motion; and one or more positive Lyapunov exponents indicate the presence of chaotic motion.

In 1979, Kaplan and Yorke [46] proposed that the fractal dimension of the attractors can be associated with the Lyapunov spectrum, i.e.,

$D_{\mathrm{L}}=j+\frac{\sum_{i=1}^{j} \lambda_{i}}{\lambda_{j+1}}$

where the Lyapunov exponents follow the order $\lambda_{1}>$ $\lambda_{2}>\lambda_{3}>\lambda_{4}>\lambda_{5}$ and $j$ is the largest integer such that $\lambda_{1}+\cdots+\lambda_{j}>0$.

Figure 10 shows the bifurcation diagram and the corresponding Lyapunov exponent spectrum and Lyapunov dimension graph in the case of $C_{M 0}=0.03$ and $\phi_{M}=200^{\circ}$; the period-doubling bifurcation transition occurs with the increase of parameter $\dot{\phi}_{\mathrm{ss}}$. The cases with parameter $\dot{\phi}_{\mathrm{Ss}}$ varying from 1.230 to 1.282 , the largest Lyapunov exponents are all very close to zero, the coning motion of rolling projectile is periodic; as parameter $\dot{\phi}_{\mathrm{ss}}$ is further increased to 1.314 , the largest Lyapunov exponents are positive; the periodic behavior of coning motion becomes irregular and turns into chaotic motion. After the chaotic motion, the response of the rolling projectile in coning motion returns back 


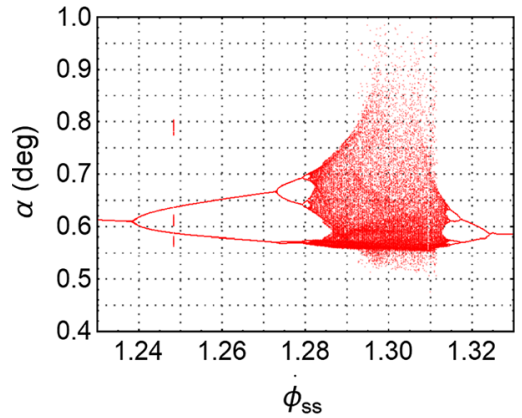

(a)

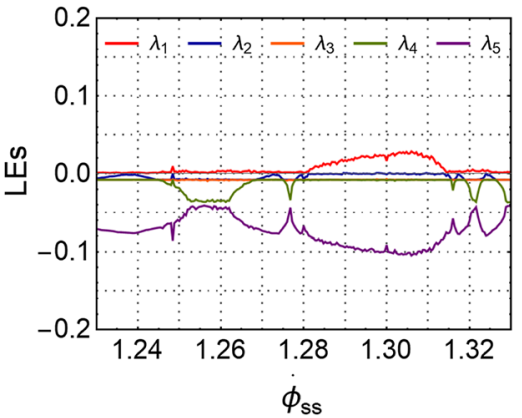

(b)

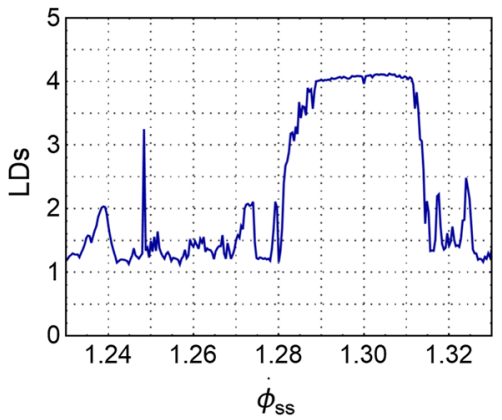

(c)

Fig. 10 Route to chaos $\left(C_{M 0}=0.03\right)$ : a bifurcation diagram; b Lyapunov exponent spectrum; and $\mathbf{c}$ Lyapunov dimension

Fig. 11 Periodic motion $\left(\dot{\phi}_{\mathrm{ss}}=1.15\right):$ a the time response; $\mathbf{b}(\alpha, \beta)$ phase portrait; $\mathbf{c}$ the Poincaré section; and $\mathbf{d}$ the power spectrum

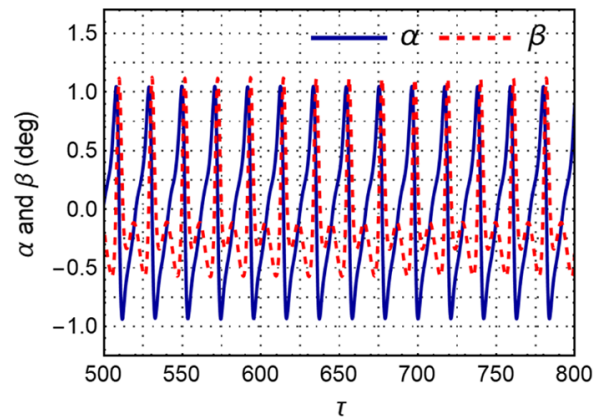

(a)

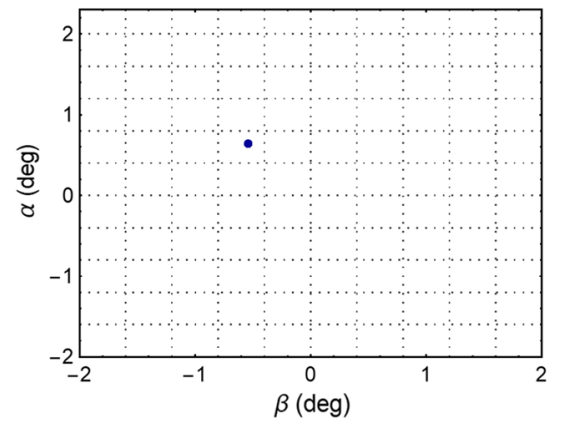

(c)

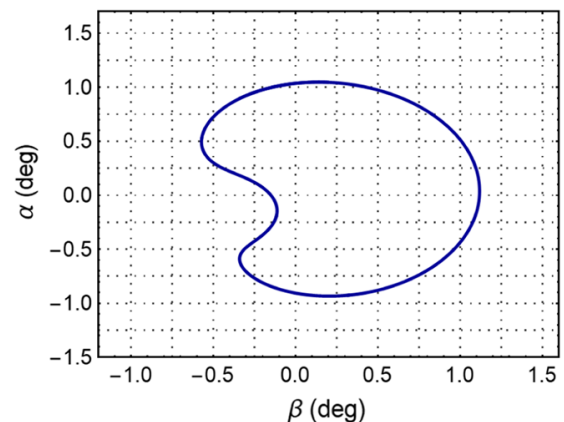

(b)

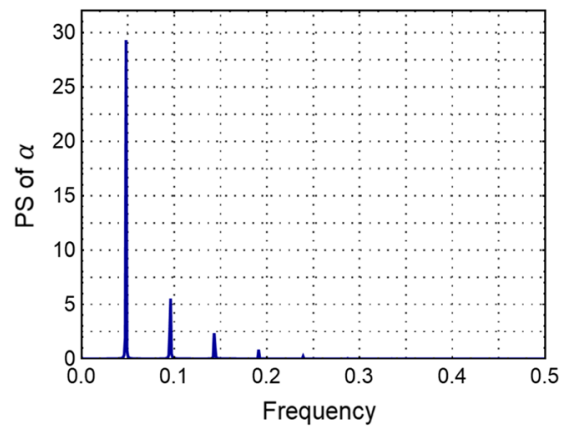

(d) to periodic motion through a reversed bifurcation as shown in Fig. 10a.

\subsection{Numerical simulation and results analysis}

The characteristic dynamical behavior of a rolling projectile in coning motion is investigated with varying parameter $\dot{\phi}_{\mathrm{ss}}$, while the asymmetric parameters are kept constants at $C_{M 0}=0.03$ and $\phi_{M}=200^{\circ}$. The time responses, the phase portraits, the power spectra, and the Poincaré sections are separately shown in
Figs. 11, 12, 13, 14, 15, 16, 17 and 18. Various types of coning motion for rolling projectiles with varying parameter $\dot{\phi}_{\mathrm{ss}}$, including regular and chaotic motion, are distinguished by these figures. The Poincare section is defined as $\Sigma^{+}=\left\{\left(\frac{v}{V}, \frac{\dot{v}}{V}, \frac{w}{V}, \frac{\dot{w}}{V}, \dot{\phi}\right) \mid \dot{\phi}=1.2\right\}$.

When $\dot{\phi}_{\text {ss }}$ is small enough, the coning motion of rolling projectiles experiences periodic motion. For $\dot{\phi}_{\mathrm{ss}}=1.15$, the time response, the phase portrait, the power spectrum, and the Poincaré section are depicted in Fig. 11. The phase trajectory has a closed orbit in evidence (Fig. 11b), the system's fundamental frequency $f$ and its harmonics can be observed in the power spec- 
Fig. 12 Period-doubling bifurcation $\left(\dot{\phi}_{\mathrm{ss}}=1.25\right)$ : a the time response; $\mathbf{b}(\alpha, \beta)$ phase portrait; $\mathbf{c}$ the

Poincaré section; and $\mathbf{d}$ the power spectrum

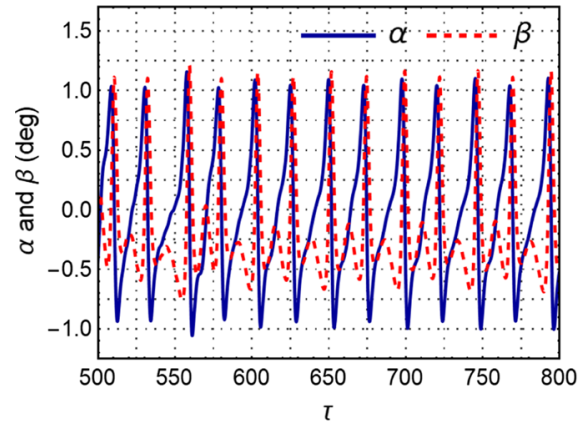

(a)

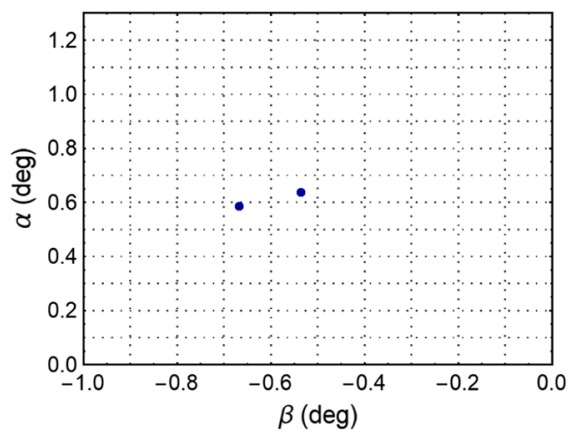

(c)

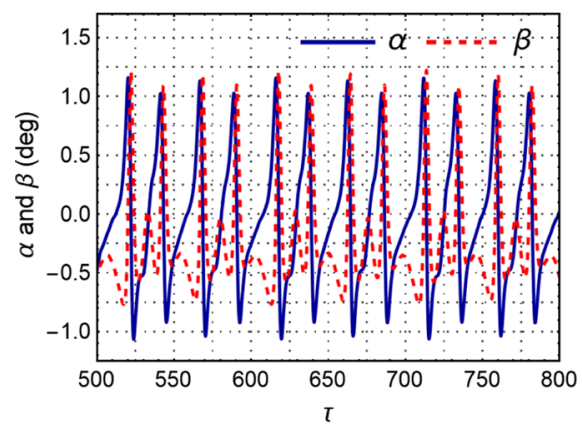

(a)

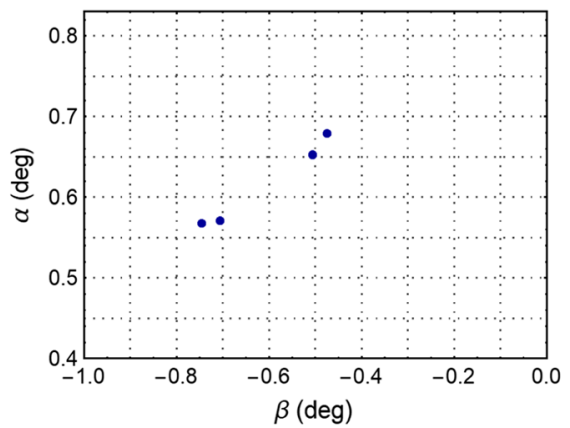

(c)

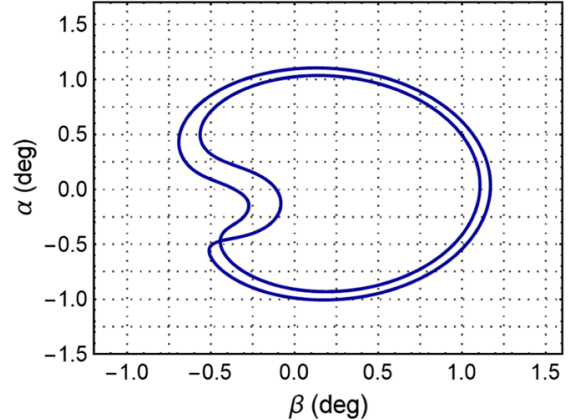

(b)

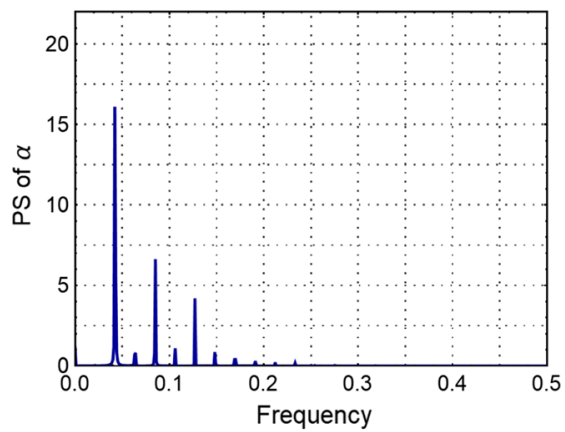

(d)

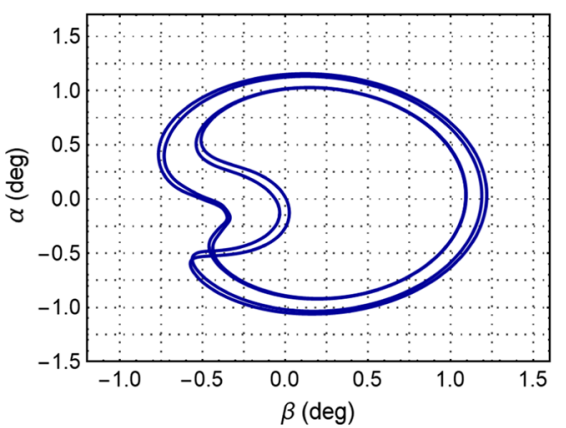

(b)

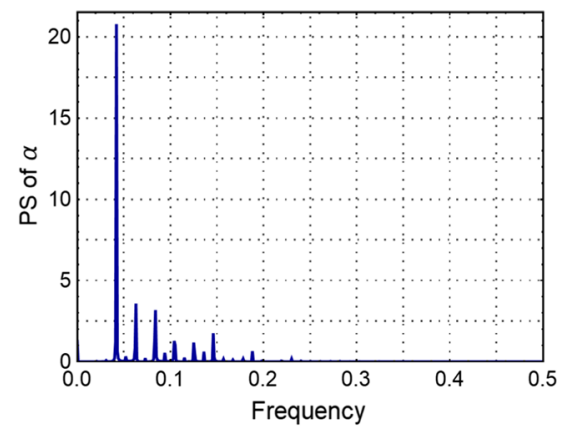

(d) 
Fig. 14 Period-doubling bifurcation $\left(\dot{\phi}_{\mathrm{ss}}=1.28\right)$ : a the time response; $\mathbf{b}(\alpha, \beta)$ phase portrait; $\mathbf{c}$ the

Poincaré section; and $\mathbf{d}$ the power spectrum

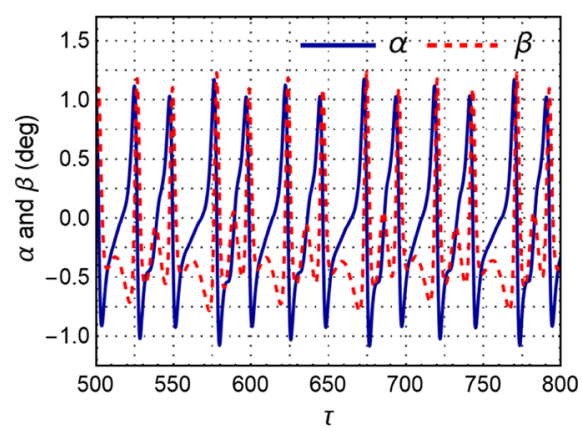

(a)

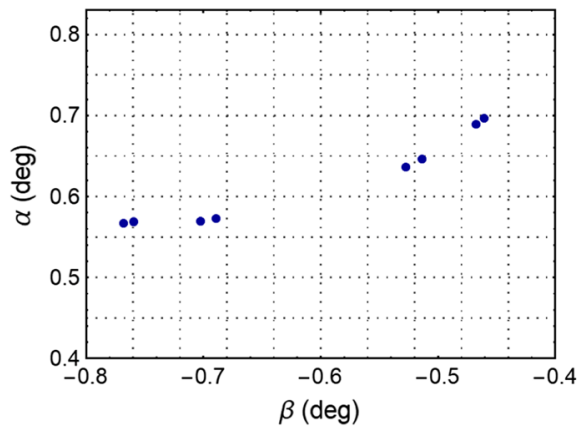

(c)

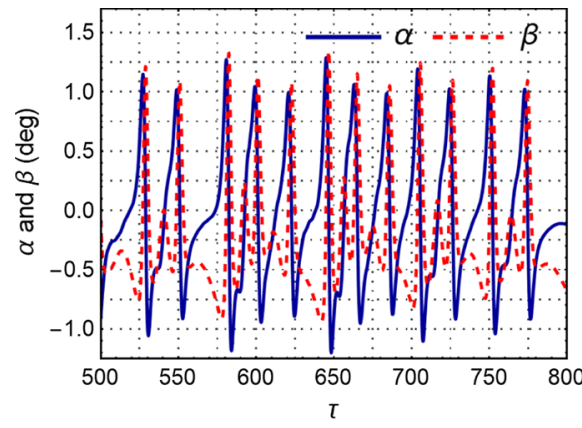

(a)

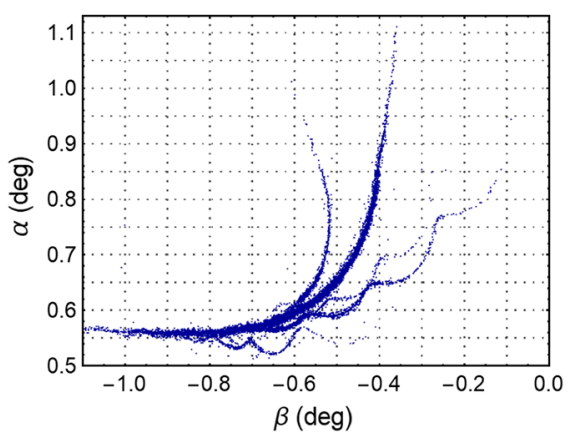

(c)

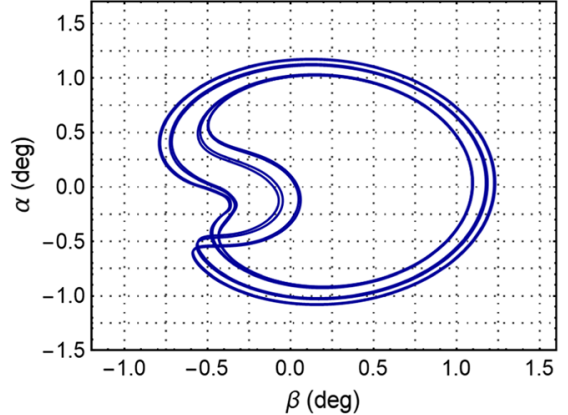

(b)

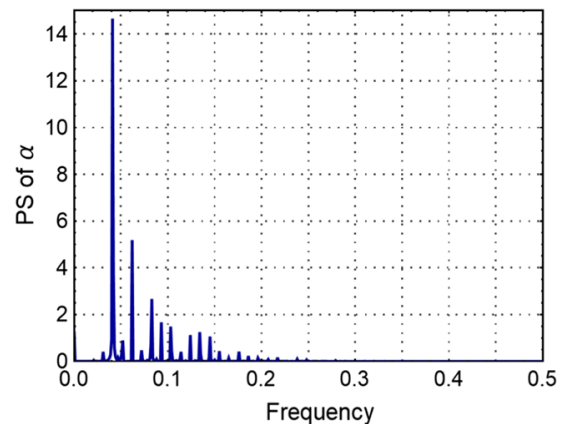

(d)

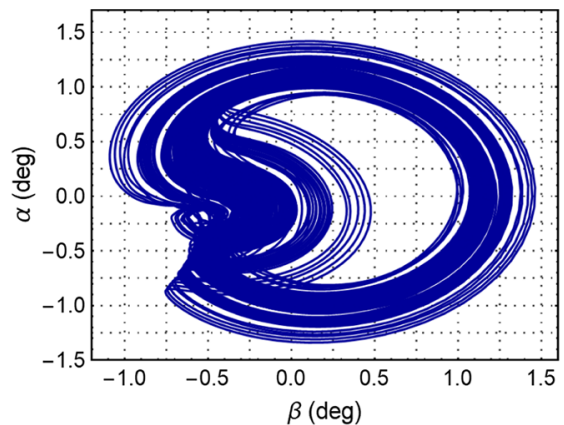

(b)

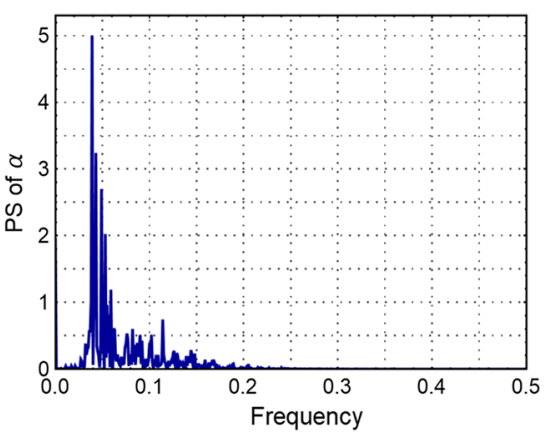

(d) 
Fig. 16 Inverse period-doubling bifurcation $\left(\dot{\phi}_{s s}=1.315\right):$ a the time response; $\mathbf{b}(\alpha, \beta)$ phase portrait; $\mathbf{c}$ the Poincaré section; and $\mathbf{d}$ the power spectrum

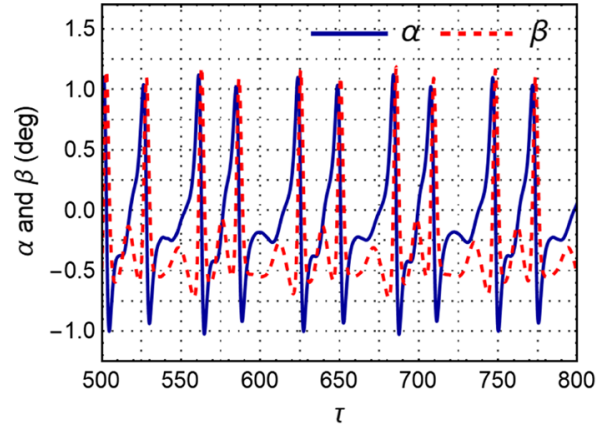

(a)

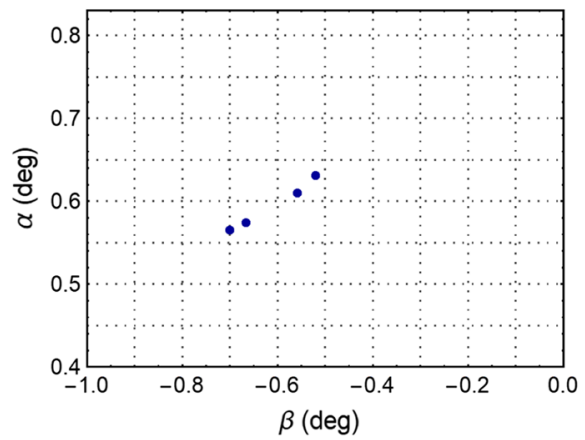

(c)

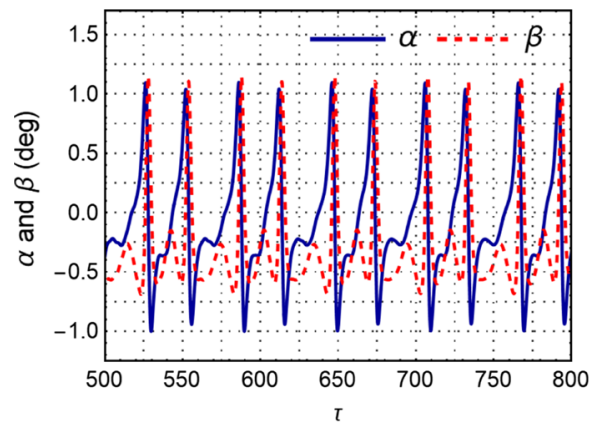

(a)

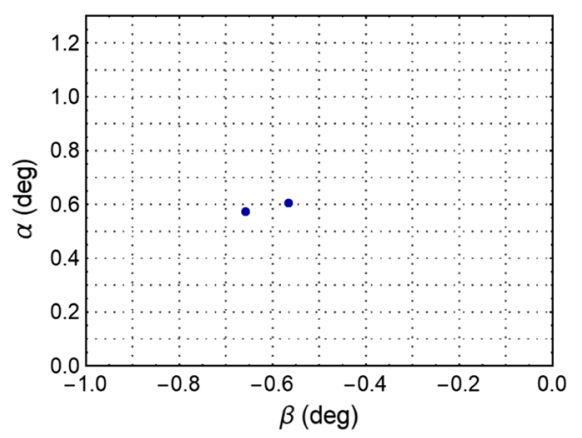

(c)

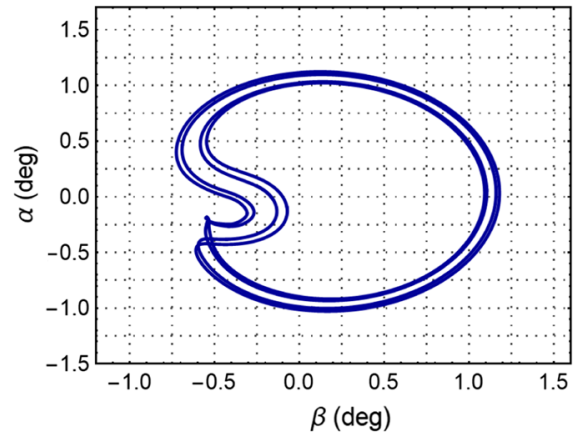

(b)

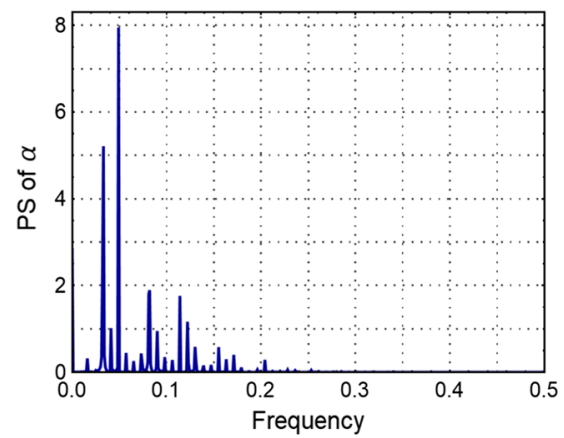

(d)

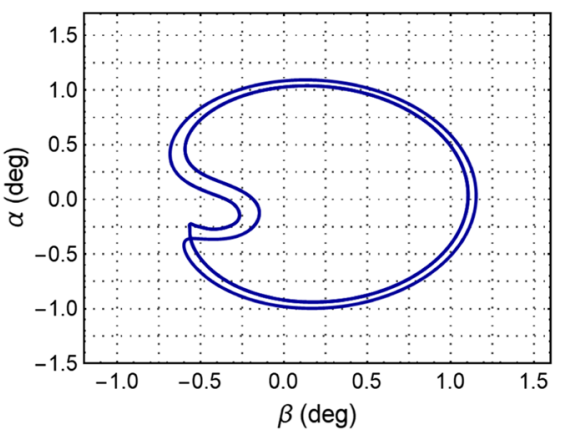

(b)

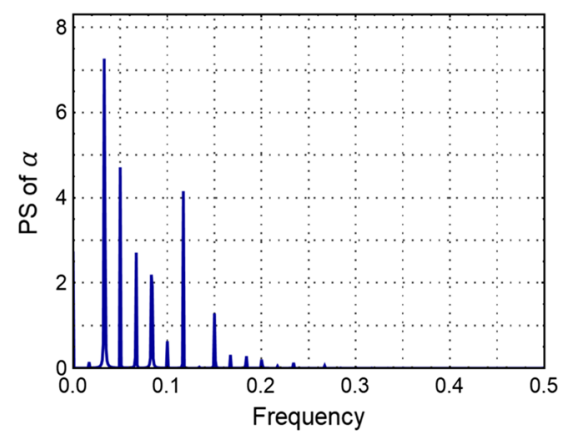

(d) 
Fig. 18 Inverse period-doubling bifurcation $\left(\dot{\phi}_{\mathrm{ss}}=1.33\right):$ a the time response; $\mathbf{b}(\alpha, \beta)$ phase portrait; $\mathbf{c}$ the Poincaré section; and $\mathbf{d}$ the power spectrum

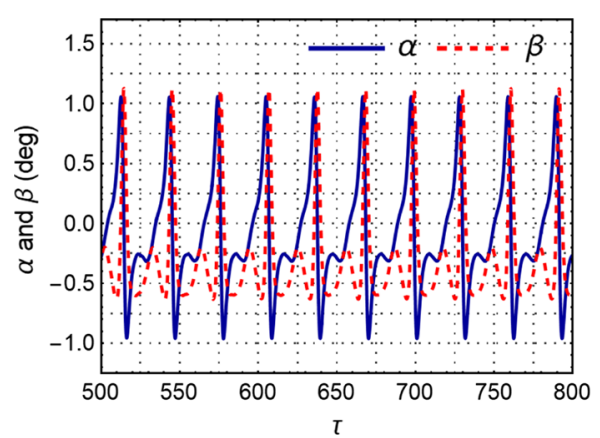

(a)

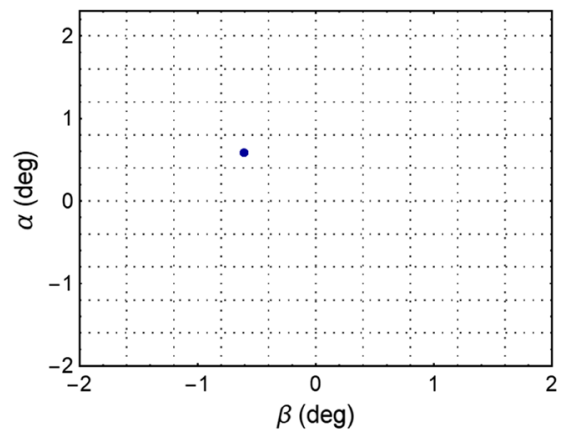

(c)

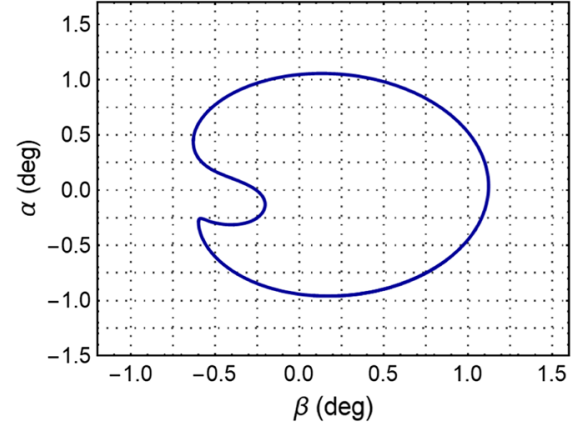

(b)

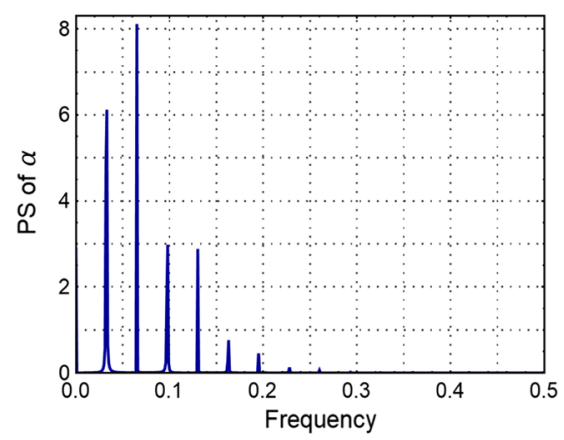

(d)

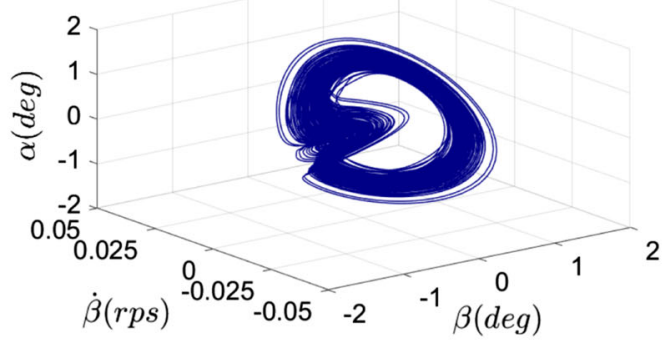

(a)

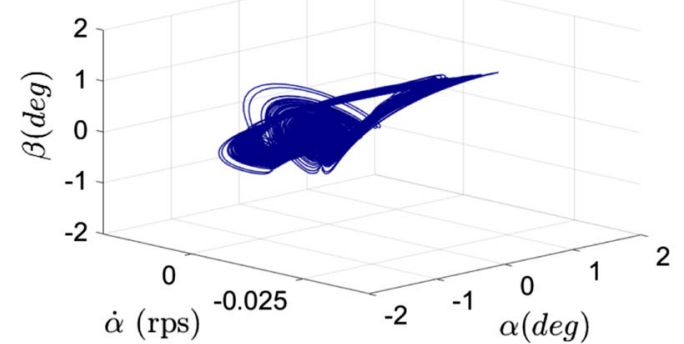

(b)

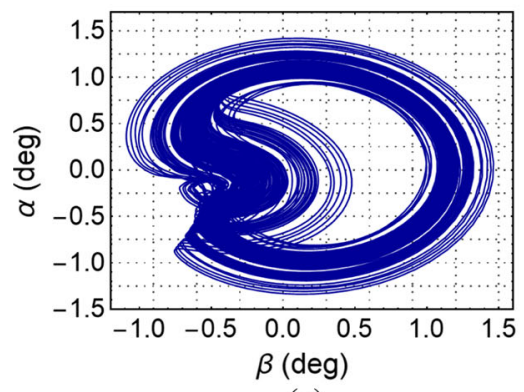

(c)

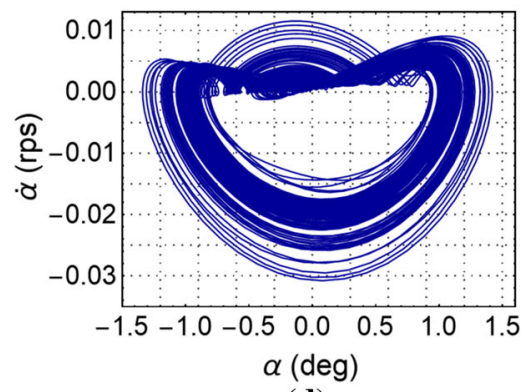

(d)

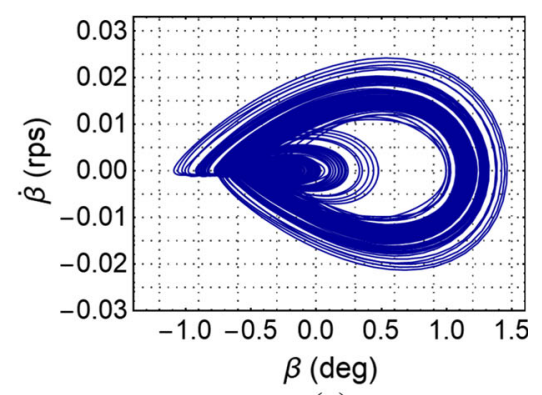

(e)
Fig. 19 Chaotic phase trajectories of rolling projectile with the initial condition $\{0.1,0,0.4,0,0\})$ : a three-dimensional subspaces $(\beta, \dot{\beta}, \alpha)$; b three-dimensional subspaces $(\alpha, \dot{\alpha}, \beta)$; c two-dimensional subspaces $(\beta, \alpha)$; $\mathbf{d}$ two-dimensional subspaces $(\alpha, \dot{\alpha})$; and e two-dimensional subspaces $(\beta, \dot{\beta})$ 
Fig. 20 Sensitivity to initial conditions $(0.1,0,0.4,0,0)$ and $(0.1,0,0.4+$ $\left.10^{-4}, 0,0\right)$ : a angle of attack $\alpha$ and $\mathbf{b}$ angle of sideslip $\beta$

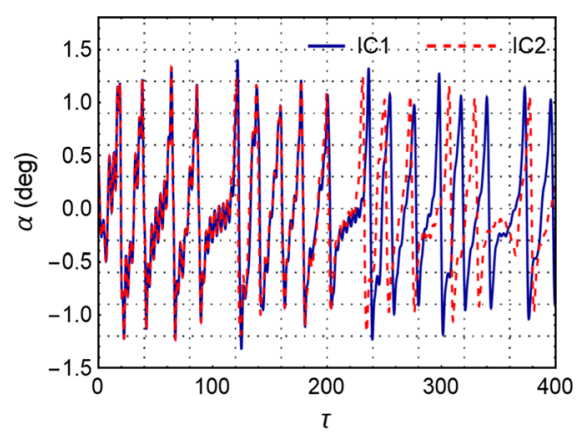

(a)

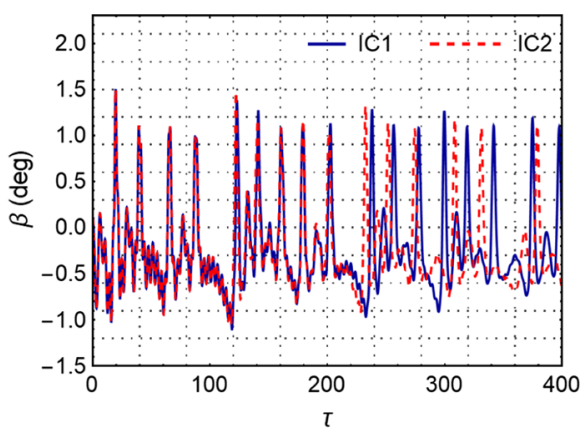

(b) trum (Fig. 11d) so that the motions are sub-harmonic resonance responses.

Varying $\dot{\phi}_{\text {ss }}$ may result in a series of period-doubling bifurcation for the coning motion of rolling projectiles. For $\dot{\phi}_{\mathrm{ss}}=1.25, \dot{\phi}_{\mathrm{ss}}=1.275$ and $\dot{\phi}_{\mathrm{ss}}=1.28$, the time responses, the phase portraits, the power spectra, and the Poincare sections are, respectively, displayed in Figs. 12, 13 and 14. It may be observed from Fig. 10 that the first bifurcation happened at $\dot{\phi}_{\mathrm{ss}}=1.238$, and is well maintained to $\dot{\phi}_{\mathrm{ss}}=1.273$. The splitting of the trajectories can be seen from these figures. From Fig. 12d, it can be seen that the power spectrum (corresponds to Fig. 11d) indicates that the bifurcation is accompanied by new spectral lines, which show that the period-doubling of the system has occurred now. Increasing $\dot{\phi}_{\mathrm{ss}}$ further results in the repetition of the previous pattern as shown in Figs. 13d and 14d; each previous phase portrait is divided into two trajectories (Figs. 13b, 14b) and each previous Poincaré map point is divided into two points (Figs. 13c, 14c).

For relatively large $\dot{\phi}_{\mathrm{ss}}$, regular motion gradually disappears and chaotic motion occurs in the coning motion of rolling projectile, the time response, the phase portrait, the power spectrum, and the Poincaré section are shown in Fig. 15. Contrasting to the Poincaré section for the periodic motion of system from Figs. 11c, 12c, 13c and $14 \mathrm{c}$, for the chaotic motion (Fig. 15c), the points fill an area in an obviously random way. In the power spectrum shown in Fig. 15d, it can be seen that the spectrum is continuous, which consists of some dominant peaks and these dominant peaks are surrounded by a lot of grass-like components.

For the further increase of $\dot{\phi}_{\mathrm{ss}}$, the inverse cascade of bifurcation can be distinguished. The time responses, the phase portraits, the power spectra, and the Poincaré maps are shown in Figs. 16, 17 and 18. Starting from the

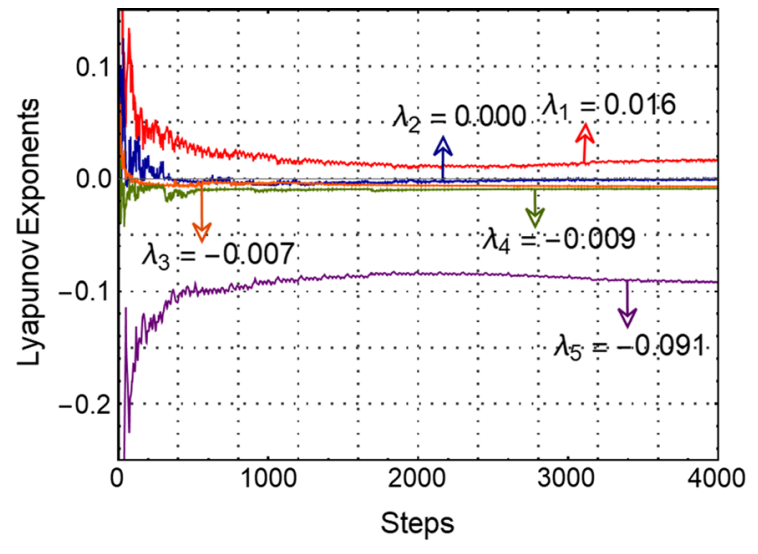

Fig. 21 Lyapunov exponents of rolling projectile system for the fixed parameter values $\dot{\phi}_{\mathrm{ss}}=1.3, C_{M 0}=0.03$ and $\phi_{M}=200^{\circ}$

chaos motion at $\dot{\phi}_{\mathrm{ss}}=1.314$, the change of parameter $\dot{\phi}_{\text {ss }}$ would cause the inverse bifurcations, jumping to the limit cycle.

For further view of the coning motion of rolling systems, Fig. 19 shows the chaotic trajectory in threedimensional subspaces $(\beta, \dot{\beta}, \alpha)$ and $(\alpha, \dot{\alpha}, \beta)$, twodimensional subspaces $(\beta, \alpha),(\alpha, \dot{\alpha})$ and $(\beta, \dot{\beta})$ for the fixed parameter $\dot{\phi}_{\mathrm{ss}}=1.33, C_{M 0}=0.03$ and $\phi_{M}=$ $200^{\circ}$. Meanwhile, the chaotic motion of coning motion for the system is also investigated by sensitivity to initial conditions as shown in Fig. 20. From Fig. 19, it can be observed that when the coning motion of rolling projectiles appears as chaotic motion, it is neither irregular nor repeated, and for time response, two trajectories are coincident at first; then they are completely independently divided with each other although they start with very near initial conditions of $(0.1,0,0.4,0,0)$ and $\left(0.1,0,0.4+10^{-4}, 0,0\right)$. The Lyapunov exponents are 0.016, 0.00, - 0.007, - 0.009, - 0.091; Fig. 21 shows the Lyapunov exponent spectrum. 
Through above analysis, it can be found that chaotic motion may occur by period-doubling bifurcation transition for the coupled angular motion and rolling motion that accounts for nonlinear aerodynamics. Chaotic motion increases the uncertainty of the system, and the coning motion cannot be predicted; hence, the occurrence of chaotic motion should be avoided as much as possible. In the design of missile control system, the analysis results of this paper are of important reference and guiding significance for the selection and design of system parameters in control design of rolling projectile systems with nonlinear aerodynamics.

\section{Conclusions}

Due to the effect of out-of-plane moment induced by the rotation of a projectile body, the rolling projectile not only rolls around its longitudinal axis continuously, but also exhibits coning motion of projectile body around the velocity vector. In this paper, the nonlinear dynamical model developed for the coning motion of a rolling projectile with slight configurational asymmetries subjected to both nonlinear aerodynamics and roll orientation-dependent aerodynamics can also be applied to describe high angle of attack in coning motion. Numerical continuation method is applied to locate the dynamically stable and unstable regions, and to determine the critical values of the bifurcation parameters trigging the qualitative change in the features of coning motion patterns, reflecting the dependence of various coning motion patterns on parameters. Period-doubling bifurcation to chaotic motion is discovered in the proposed system by Lyapunov exponent analysis and bifurcation diagrams revealing how a regularly behaving coning motion becomes chaotic motion with the variation of system parameters. Numerical methods including time response, phase trajectory, Poincaré section and power spectrum are employed to demonstrate the characteristic nonlinear dynamical behavior appearing in the coning motion of asymmetric rolling projectiles. Researches presented reveal the abundant dynamic properties of periodic orbits and chaos occurring in asymmetric rolling projectile motions with nonlinear aerodynamics. This study will contribute much to an in-depth understanding for the nonlinear dynamic characteristics of asymmetric rolling projectiles and thus provide significant guidance in control design for the nonlinear projectile motion.
Acknowledgements Our special thanks go to Arun K. Banerjee for his valuable suggestions to this paper. The work presented in this paper was supported by National Natural Science Foundation of China (Grant Nos. 11472041, 11532002, 11772049).

\section{Compliance with ethical standards}

Conflict of interest The authors declare no conflict of interest.

\section{References}

1. Nicolaides, J.D.: On the free flight motion of missiles having slight configurational asymmetries. U. S. Army Ballistic Research Lab, Aberdeen Proving Ground, MD, BRL Rept. 858, 1952, AD 26405; also, Institute of Aeronautical Sciences Paper 395 (1952)

2. Nicolaides, J.D.: Two nonlinear problems in the flight dynamics of modern ballistic missiles. Report 59-17, Institute of the Aeronautical Sciences (1959)

3. Price Jr., D.A.: Sources, mechanisms and control of roll resonance phenomena for sounding rockets. J. Spacecr. Rockets 4, 1516-1525 (1967)

4. Murphy, C.H.: Some special cases of spin-yaw lock-in. J. Guidance Control Dyn. 12, 771-776 (1989)

5. Ananthkrishnan, N., Raisinghani, S.C.: Steady and quasisteady resonant lock-in of finned projectiles. J. Spacecr. Rockets 29, 692-696 (1992)

6. Ananthkrishnan, N., Raisinghani, S.C., Pradeep, S.: Transient resonance of rolling finned projectiles. Proc. Inst. Mech. Eng. Part G J. Aerosp. Eng. 213, 97-103 (1999)

7. Sharma, A., Ananthkrishnan, N.: Passage through resonance of rolling finned projectiles with center-of-mass offset. J. Sound Vib. 239, 1-17 (2001)

8. Pepitone, T., Jacobson, I.: Resonant behavior of a symmetric missile having roll orientation-dependent aerodynamics. J. Guidance Control Dyn. 1, 335-339 (1978)

9. Murphy, C.H., Mermagen, Wm H.: Spin-yaw lock in of a rotationally symmetric missile. J. Guidance Control Dyn. 32, 377-382 (2009)

10. Li, D., Chang, S., Wang, Z.: Analytical solutions and a novel application: insights into spin-yaw lock-in. J. Guidance Control Dyn. 40, 1472-1481 (2017)

11. Morote, J.: Resonant lock-in of unguided rockets having nonlinear aerodynamic properties. In: AIAA Paper 2006, p. 830 (2006)

12. Morote, J., Liaño, G., Jiménez, J.: Nonlinear rolling motion of triform finned missiles. J. Spacecr. Rockets 54, 459-470 (2017)

13. Liaño, G., Castillo, J.L., García-Ybarra, P.L.: Nonlinear model of the free-flight motion of finned bodies. Aerosp. Sci. Technol. 39, 315-324 (2014)

14. Liaño, G., Castillo, J.L., García-Ybarra, P.L.: Steady states of the rolling and yawing motion of unguided missiles. Aerosp. Sci. Technol. 59, 103-111 (2016)

15. Iñarrea, M.: Chaos and its control in the pitch motion of an asymmetric magnetic spacecraft in polar elliptic orbit. Chaos Solitons Fractals 40, 1637-1652 (2009)

16. Faramin, M.: Chaotic attitude analysis of a satellite via Lyapunov exponents and its robust nonlinear control subject to 
disturbances and uncertainties. Nonlinear Dyn. 83, 361-374 (2015)

17. Abdelaziz, Y.A., Shoaib, M.: Numerical analysis of the attitude stability of a charged spacecraft in the Pitch-Roll-Yaw directions. Int. J. Aeronaut. Space Sci. 15, 82-90 (2014)

18. Liu, Y.Z., Chen, L.Q.: Chaos in Planar Attitude Motion of Spacecraft. Springer, Berlin (2013)

19. Nicolaides, J.D.: A review of some recent progress in understanding catastrophic yaw. Rept. 551, AGARD (1966)

20. Tanrikulu, O.: Limit cycle and chaotic behavior in persistent resonance of unguided missiles. J. Spacecr. Rockets 36, 859865 (1999)

21. Liu, Y.Z., Chen, L.Q.: Chaos in Attitude Dynamics of Spacecraft. Tsinghua University Press, Springer (2012)

22. Nayfeh, A.H., Balachandran, B.: Applied Nonlinear Dynamics: Analytical, Computational, and Experiment Methods. Wiley, New York (1995)

23. Moon, F.C.: Chaotic and Fractal Dynamics: An Introduction for Applied Scientists and Engineers. Wiley, New York (1992)

24. Alligood, K.T., Sauer, T.D., Yorke, J.A.: Chaos: An Introduction to Dynamical Systems. Springer, New York (1996)

25. Ott, E.: Chaos in Dynamical Systems, 2nd edn. Cambridge University Press, Cambridge (2002)

26. Grebogi, C., Ott, E., Yorke, J.A.: Are three-frequency quasiperiodic orbits to be expected in typical dynamical systems? Phys. Rev. Lett. 51, 339-342 (1983)

27. Grebogi, C., Ott, E., Yorke, J.A.: Crises, sudden changes in chaotic attractors and transient chaos. Phys. D 7, 181-200 (1983)

28. Luliana, O., Gerhard, D.: A period doubling route to spatiotemporal chaos in a system of Ginzburg-Landau equations for nematic electroconvection. Discrete Contin. Dyn. Syst. B 24, 273-296 (2019)

29. Lin, D.C., Oguamanam, D.C.D.: A numerical study of the dynamics of three-mass system on frictional tracks. Nonlinear Dyn. 94, 2047-2058 (2018)

30. Yang, Y., Ren, X., Qin, W., et al.: Analysis on the nonlinear response of cracked rotor in hover flight. Nonlinear Dyn. 61, 183-192 (2010)

31. Luo, A.C.J., Xing, S.Y.: Bifurcation trees of period-3 motions to chaos in a time-delayed Duffing oscillator. Nonlinear Dyn. 88, 2831-2862 (2017)

32. Wiggins, S.: Introduction to Applied Nonlinear Dynamical Systems and Chaos. Springer, New York (2013)

33. Beyn, W.J., Champneys, A., Doedel, E.J., Kuznetov, Y.A., Govaerts, W., Sandstede, B.: Numerical continuation and computation of normal forms. In: Fiedler, B. (ed.) Handbook of Dynamical Systems, vol. 2, pp. 149-219. Elsevier, Amsterdam (2001)

34. Beyn, W.J., Champneys, A., Doedel, E.J., Kuznetov, Y.A., Govaerts, W., Sandstede, B.: Numerical continuation and computation of normal forms. In: Fiedler, B. (ed.) Handbook of Dynamical Systems, vol. 2, pp. 149-219. Elsevier, Amsterdam (2001)

35. Léger, S., Deteix, J., Fortin, A.: A Moore-Penrose continuation method based on a Schur complement approach for nonlinear finite element bifurcation problems. Comput. Struct. 152, 173-184 (2015)

36. Kuznetsov, Y.A.: Elements of Applied Bifurcation Theory, 2nd edn. Springer, New York (1998)

37. Yang, S.X., Zhao, L.Y., Yan, X.Y.: Dynamic Stability of Spinning Missiles. National Defense Industry Press, Beijing (2014)

38. Han, Z.P., Chang, S.J., Shi, J.G.: Nonlinear Motion Theory of Projectile and Rocket. Beijing Institute of Technology Press, Beijing (2016)

39. Sun, H.D., Yu, J.Q., Zhang, S.Y.: Bifurcation analysis of the asymmetric rolling missiles. Proc. Inst. Mech. Eng. Part G J. Aerosp. Eng. 230, 1273-1283 (2016)

40. Kuznetsov, N.V., Alexeeva, T.A., Leonov, G.A.: Invariance of Lyapunov exponents and Lyapunov dimension for regular and irregular linearizations. Nonlinear Dyn. 85, 195-201 (2016)

41. Vallejo, J.C., Sanjuan, M.A.F.: Predictability of Chaotic Dynamics. Springer, Berlin (2017)

42. Wiggers, V., Rech, P.C.: Chaos, periodicity, and quasiperiodicity in a radio-physical oscillator. Int. J. Bifurc. Chaos 27, 22 (2017)

43. Benettin, G., Galgani, L., Giorgilli, A., et al.: Lyapunov characteristic exponents for smooth dynamical systems and for Hamiltonian systems; a method for computing all of them. Part 1: theory. Meccanica 15, 9-20 (1980)

44. Benettin, G., Galgani, L., Giorgilli, A., et al.: Lyapunov characteristic exponents for smooth dynamical systems and for Hamiltonian systems; a method for computing all of them. Part 2: numerical application. Meccanica 15, 21-30 (1980)

45. Kuznetsov, N.V.: The Lyapunov dimension and its estimation via the Leonov method. Phys. Lett. A 380, 2142-2149 (2016)

46. Kaplan, J., Yorke, J.: Chaotic behavior of multidimensional difference equations. In: Peitgen, H.O., Walther, H.O. (eds.) Functional Differential Equations and Approximation of Fixed Points. Springer, New York (1979)

Publisher's Note Springer Nature remains neutral with regard to jurisdictional claims in published maps and institutional affiliations. 